\section{IJ§ER}

ISSN: 2149-5939
International Journal of Social Sciences and Education Research

Online, https://dergipark.org.tr/tr/pub/ijsser

Volume: 7(4), 2021

\title{
Comparative analysis of estimated market values of companies: Forbes 2000 and BIST 388 company example
}

\author{
Çağnı Köroğlu ${ }^{1}$, Cahit İncioğlu² and Zeynep Aydın ${ }^{3}$ \\ ${ }^{1}$ Assoc. Prof. Dr., Aydın Adnan Menderes Un., Nazilli FEAS, Nazilli, Aydın, Turkey, cagri.koroglu@adu.edu.tr ORCID: https://orcid.org/0000- \\ 0003-4073-1847 \\ ${ }^{2}$ Ph.D. Student, Aydın Adnan Menderes Un., Business, Aydın, Turkey, cahitincioglu@gmail.com, ORCID: https://orcid.org/000-0003-2449- \\ 5346 \\ ${ }^{3}$ Postgraduate Student, Aydin Adnan Menderes Un., Accounting and Finance, Aydın, Turkey, zynpayydin@gmail.com, ORCID: https://or- \\ cid.org/0000-0002-3358-0000
}

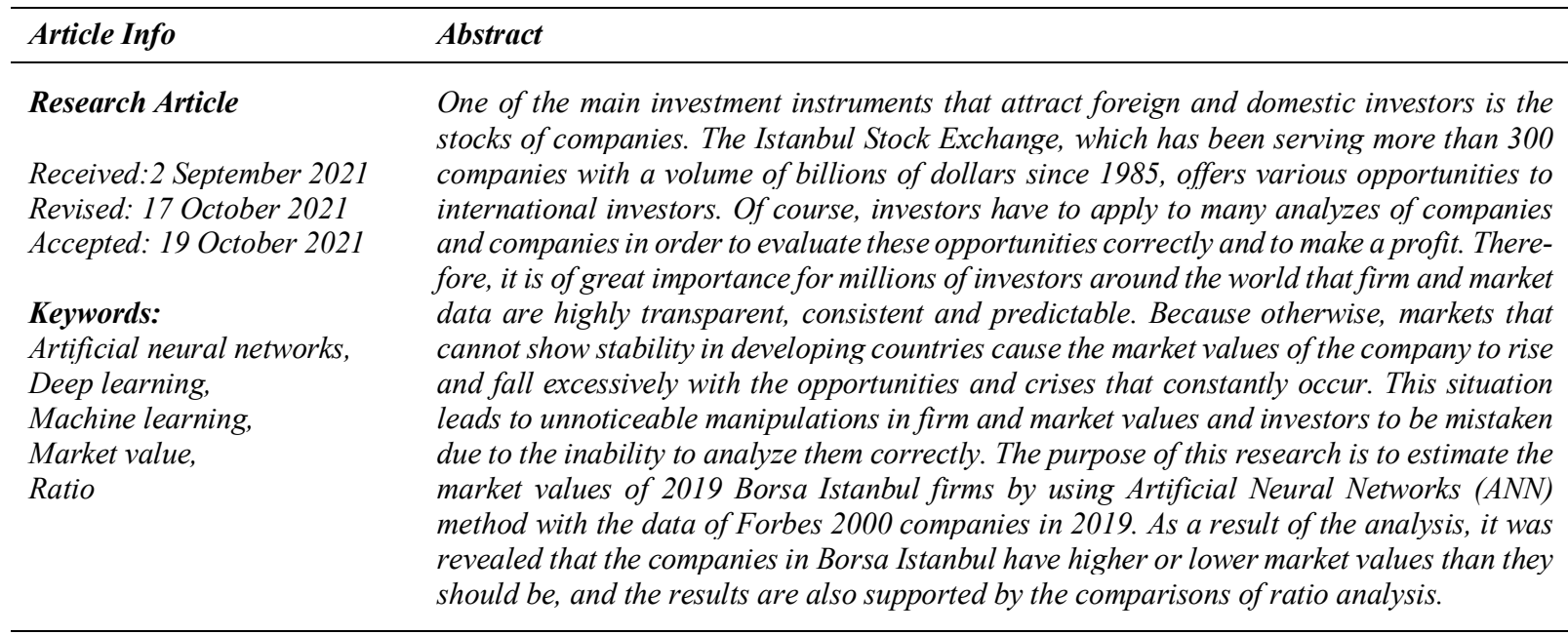

\section{Introduction}

Equity markets are a public market in which company shares are traded at an agreed price and offer investors the opportunity to share the company's profits based on the amount of ownership (Barnes, 2016). Investors need to analyze the market and the company correctly in order to predict these ups and downs. However, manipulations that may arise with high competition can cause competition and mislead investors (Aggarwal and Wu, 2003). Future price predictions of stocks can also be made by methods such as artificial neural networks (Khan etc., 2011). In addition, there are many studies showings that the values of stocks offered for sale during the global crisis decreased to lower levels than expected (Boudriga and Ghachem, 2016). In this risky conjuncture, one of the most concrete methods of providing information about the business performance and economic variables of companies is ratio analysis (Drake and Fabozzi, 2012). However, for these analyzes to yield clear results, company data should be compared with companies in global markets and unreliable markets or companies should be distinguished from others. Ratio analysis is one of the main indicators by which investors can predict the financial performance of companies they want to buy stocks (Nikolai etc. 2009). In these analyzes, variables such as the sales amount, profit, assets and market values of the companies are used.

In this research, the estimated market values of Borsa Istanbul companies, which are a platform where international investors can evaluate their money, were reached and compared with the real market values. Based on the optimum sales, profit, assets and market values of the best Forbes 2000 companies in 2019 the sales, profit and asset data of the companies in Borsa Istanbul were analyzed and compared with the artificial neural networks and

* Ethics Committee Approval is not required for this study. All responsibility belongs to the researchers.

To cite this article: Köroğlu, Ç., İncioğlu, C. \& Aydın, Z. (2021). Comparative analysis of estimated market values of companies: Forbes 2000 and BIST 388 company example. International Journal of Social Sciences and Education Research, 7 (4), 369-390. DOI: https://doi.org/10.24289/ijsser.990376 
the estimated market values of Borsa Istanbul. In this research, in which the data of hundreds of companies were used, artificial neural networks provided great convenience. Contrary to many operations and time loss in traditional methods, artificial neural networks, which are a modern method, both save time and unlike operations that can be complex, thanks to the database, practicality has been gained and the probability of error in this direction has decreased, which makes the method more valuable.

\section{Factors affecting the market value of companies and analysis}

\subsection{Factors affecting market value}

Market value, which is of great importance for both investors and company managers, can be calculated using the main factors that interact economically with each other such as assets, income, profit and many other variables such as debts, interests, savings and cash flows (Pareja \& Tham, 2009). For example; It can be said that assets and income can affect the tax amount, and the tax amount can affect the amount of profit, and in this case, all variables that affect the cash flow affect the market value of the company.

\subsubsection{Property assets}

Assets represent the valuable things owned by a household, firm or government. It refers to the assets of the company in all items such as material, immaterial, trade receivables, stocks and cash flow. Increase or decrease of these assets have great effects on the calculation of company balance sheet (Deiter, 1991). Companies pay taxes according to the results in their balance sheets, and companies whose balance sheets change can increase or decrease their market value by changing their cash flow costs in both tax and other investment instruments (Machado \& Medeiros, 2013). For that reason, it can be said that assets can affect company values directly or indirectly.

\subsubsection{Income}

Income is the income that a firm earns from the sale of its products or services (Sexton, 2015). The multiplication of the number of products or services sold by the selling price gives the total revenue. In addition, company income is one of the points that investors should pay attention to. Because another criterion used in market value calculations is the net return per share of the invested company (Thomas \& Gup, 2010): Market Value per share $=$ Price per share / Net income per share. But in order to find net income, all expenses must be subtracted from the income and net profit presented.

\subsubsection{Profit}

Profit refers to the excess of the business personality's equity at the end of the period over the equity at the beginning of the period. In other words, it can be defined as the increase in net assets (Kizil et al., 2013). The increase in the profit level can increase the assets and liquidity assets and this situation can increase the value of the company in the market. As the economic profit decreases or increases, the market value added, which informs about the feasibility of investment and operating processes, also changes (Peterson and Fabozzi, 1999). In short, the amount of profit affects the market value by changing both the market value and the demand for the company with the preferences of the investor. Market Value is obtained by multiplying the share price of a firm by the total number of shares (Krantz, 2015). The position of companies in the market is important for investment owners. In order to determine the position of the firms in the market, the market value must be calculated. The current position of firms in markets where competition is intense is of great importance for investors in the decision-making process (Eiteman and Stonehill, 1986).

\subsection{Financial ratio analysis methods}

Investors or managers use financial ratios to learn more about the past, future and economic potential of the company (Tracy, 2012). These ratios serve to understand information that does not appear in the data presented in the markets and serve as a provision for good or bad results about the company.

\subsubsection{Asset turnover speed}

Asset turnover is used as a measure of capital intensity or efficiency in the use of assets (Needles et al., 2013). The calculation is Net sales divided by Average total assets. Assuming that other conditions in the business remain the same, it can be said that the profitability ratios of companies with high asset turnover are also high. This ratio should be at least 1.5, companies between 2 and 4 are in good condition. The fact that this ratio is low indicates that there is idle capacity in the enterprise (Peterson, 2006). 


\subsubsection{Profitability of actives}

The profitability ratio of assets is used to evaluate to what extent a firm's ability to make a profit with respect to its total assets (Berman et al., 2008). It also specifies how it uses assets to generate profit for efficient management. Return on assets is calculated by dividing the annual earnings of a firm by total assets (Angenieux, 1964). It can be said that the higher the coefficient of the result, the more successful the companies are in making a profit.

\subsubsection{Price sales ratio}

The price selling ratio is found by proportioning the total market value to the sales. This ratio shows how much the investor pays for every $\$ 1$ profit the company generates (Brigham \& Houston, 2012). It is better for investment decisions to have a small coefficient of the ratio indicating how many times the firm value is the business volume. In order to analyze how high or low this ratio is, firstly, an average ratio should be determined by making a sectoral comparison (Giacomino \& Mielke, 1993).

\subsubsection{Share price measurement}

The high number of coefficients of the share price measurement calculated by the ratio of firm value to profit indicates that the share price is high, and the low number of times indicates that the price is low (Shim \& Siegel, 1988). It shows how many times the net profit is on an annual basis within the market value of the company (Taner \& Akkaya, 2004). This ratio is generally preferred to be low (Bhagat \& Black, 2001). At the same time, whether the ratio is low or high can be better understood by comparing it with the Price / Earnings ratio of other businesses operating in the same sector or with the average price earning ratio of the sector.

F / K (Share price measurement $)=$ Market Value / Net Profit for the Period

\section{Literature review}

The study in which Singh and Srivastava used Deep Learning (DL) for stock data prediction can be considered as one of the first analyzes on this subject, and it was revealed that the deep learning method gave better results than other neural networks (Singh \& Srivastava, 2017). In a study conducted on NSE Stock Market, 2 deep learning models were used for stock prediction and it was concluded that the deep learning method performed better than linear time series models (Hiransha etc., 2018). In a study conducted on the China Stock Exchange in 2018, a deep architecture-based model was built for stock market prediction and the use of artificial neural networks in analysis with big data was proven to be an effective method (Chen etc., 2018).

Vargas, Lima, and Evsukoff have tried to predict the movements of the Standard \& Poor index with the Deep Learning method based on the news published in financial articles, and have conducted a study that provides evidence that even words can be converted into financial predictions (Vargas etc., 2017). In a study conducted for Borsa İstanbul, the estimation of stocks was made with the help of Artificial Neural Networks and it was proved that the performances of ANN models are around 70\% more efficient than other models (Kara etc., 2011). Research by Dase \& Pawar revealed that Artificial Neural Networks (ANN) methods are useful for predicting the stock index and also have a feature that can be used to predict whether it is best to buy, hold or sell stock market shares (Dase \& Pawar, 2010).

In the study of Yetis, Kaplan and Jamshidi, ANN model was used for the estimation of stock market prices and as a result of the research; It has been stated that for individual and institutional investors, financial analysts and financial news users, anticipating the future behavior and movement of stock prices can help them to show the necessary behaviors in advance in order to gain more profit or not to lose (Yetis et al., 2014). In an application by Qiu, Song and Akagi on the Japanese Stock Exchange, estimates were made using ANN and it was concluded that not only market value but also real import and export values, discounts, interest and bond estimates could be estimated (Qiu et al., 2014). Cao, Leggio and Schniederjans made a comparison between Fama and French's model and artificial neural networks to predict the Chinese stock market, and given the decreasing availability of information in emerging markets and the sometimes questionable quality of information, investors' It has been found to be more usable to increase predictive power (Cao et al., 2005).

Zhang and Wu applied the ANN model for the estimation of the S\&P 500 index, and with different optimizations integrated into the artificial neural network, better estimates were achieved with less computational complexity in stock market forecasts (Zhang \& Wu, 2009). Patel and Yalamalle analyzed a number of stocks in the Indian Stock Exchange and it was found that the ANN technique was very useful in predicting stock prices of a particular company as well as stock indices (Patel \& Yalamalle, 2014). 


\section{Material and method}

In this study, many options of the Phyton program were used to analyze the company data in Forbes 2000 according to the characteristics of the companies in Borsa Istanbul and determine their market values. In the study, the entire data set consisting of information about 2000 companies should be analyzed and weighted in each Turkish company. In other words, for the market values entered into the program, it should compare all the data and choose the most approximate market value. Since the data set is slightly larger than the standard survey data and 2 thousand analyzes are required each time, high data analysis speed is required. Therefore, such processes may require machine learning and statistics to work together (Atalay \& Çelik, 2017): In the study, Artificial Neural Networks (ANN) method was used as one of the artificial intelligence applications.

\subsection{Artificial neural networks (ANN)}

In information technology, ANN is a hardware and / or software system modeled after the study of neurons in the human brain. ANNs are also called neural networks for short and are various deep learning technologies that fall under the umbrella of artificial intelligence or machine learning (Rouse, 2019). Neural networks method is particularly successful in detecting nonlinear patterns. Artificial neural networks, pattern recognition and optimization are also used. Artificial neural network applications include supervised and unsupervised learning (Çelik, 2018: 122). In its simplest form, the neural network is a "sensor" consisting of a single neuron. Much like biological neurons with dendrites and axons, a single artificial neuron is a simple tree structure with input nodes and a single exit node connected to each input node (Willems, 2019). The comparison of biological neuron and ANN is given below.

\section{Biological Neuron versus Artificial Neural Networks}

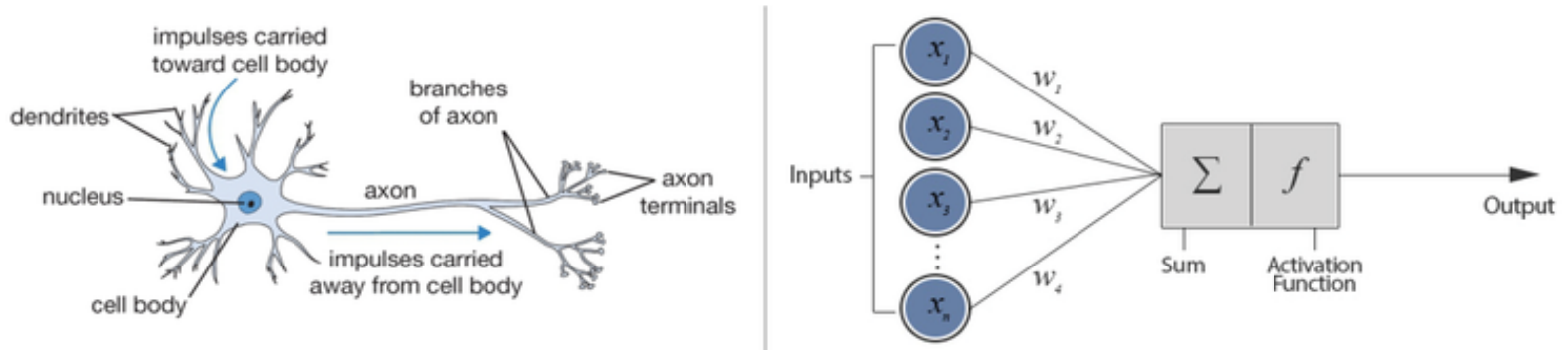

Figure 1. Comparison of Biological Neuron and ANN

Source: Willems, (2019).

As it can be seen on Figure 1, artificial neurons have six components. From left to right, these are (Willems, 2019):

1. Input nodes: Each input node is associated with a numeric value that can be any real number. Real numbers make up the full spectrum: they can be positive or negative, integers or decimals.

2. Links: Each link leaving the login node has a weight associated with it, and this can also be any real number.

3. Then all the values of the input nodes and the weights of the links are combined together: they are used as inputs for the weighted sum: or expressed in a different way,

4. This result will be the input for a transfer or activation function. In the simplest but trivial case, this transfer function will be an identity function.

$$
\begin{aligned}
& \text { Weighted total }=y=f\left(\sum_{i}^{D} \omega_{i} \cdot x_{i}\right) \\
& y=f\left(\omega_{1} \cdot x_{1}+\omega_{2} \cdot x_{2}+\cdots+\omega_{D} \cdot x_{D}\right) \\
& f(x)=x y=x x \\
& f(x)=0 x<0 \\
& f(x)=0.5 x=0 \\
& f(x)=1 x>0
\end{aligned}
$$


For sure, the output may not be a linear function: it may be a discontinuous function. Since this can cause problems in mathematical processing, the sigmoid function, which is a continuous variable, is often used. As you already know, a sigmoid function is also a logistics function. Using the logistics function provides a much simpler result.

5. As a result, you have the exit node associated with the function of the weighted sum of input nodes (such as the sigmoid function). The sigmoid function is a mathematical function that results in an "S" shaped curve mathematically as follows:

$$
S(x)=\frac{1}{1+e^{-x}}=\frac{e^{x}}{e^{x}+1}
$$

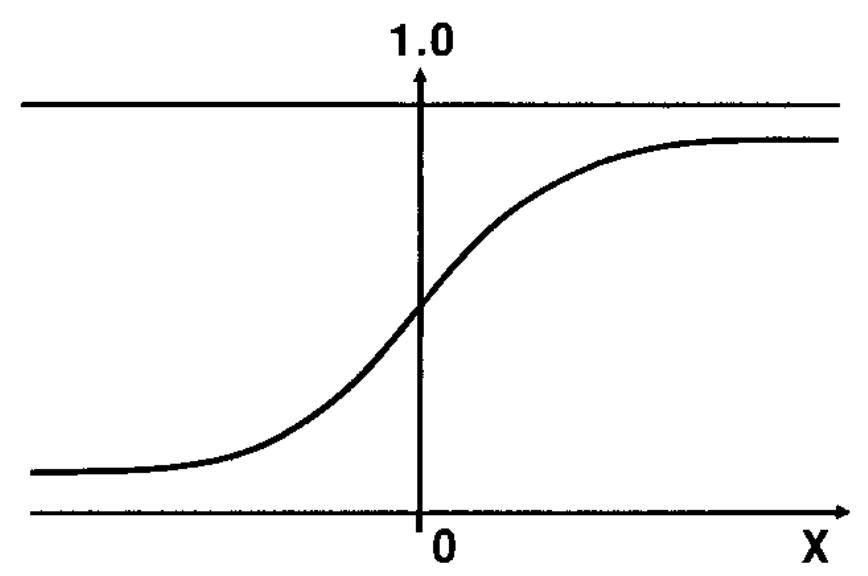

Figure 2. Sigmoid function

Source: Næs et al., (1993).

6. Finally, the sensor can give with an input node advancing to 1 permanently, a parameter called bias. The deviation value is critical because it allows you to scroll to this. Left or right, I can determine the success of your learning.

ANN usually includes a large number of processors running in parallel and arranged in layers. The first stage receives raw input information similar to the optic nerves in human visual processing. Each successive layer receives output from the layer preceding it rather than the raw input, so neurons further away from the optic nerve receive signals from those close to it. The last stage produces the output of the system. Each compute node has its own small area of information, including the rules it sees and is initially programmed or developed for itself. The layers are highly interconnected; This means that each node in layer $\mathrm{n}$ will be connected to multiple nodes in layer $\mathrm{n}-1$ (inputs) and layer $\mathrm{n}+1$ providing input data for those nodes. The output layer can have one or more nodes where the output it produces can be read (Rouse, 2019). Artificial neural networks stand out for their adaptability, which means they change themselves as they learn from initial training, and subsequent studies provide more information about the world. The most basic learning model focuses on weighting input streams; it is how each node weighs the importance of the input data from each of its entries. Inputs contributing to obtaining correct answers are heavier (Rouse, 2019).

Deep learning is a subfield of machine learning, a set of algorithms inspired by the structure and function of the human brain. These algorithms are often called Artificial Neural Networks (ANN). Deep learning is one of the hottest areas in data science, with many case studies in the fields of robotics, image recognition, and Artificial Intelligence (AI) with surprising results. One of the most powerful and easy-to-use Python libraries for developing and evaluating deep learning models is Keras; It complements the efficient numerical computing libraries Theano and TensorFlow. The advantage of this is that it basically enables us to get started with neural networks in an easy and fun way (Willems, 2019).

Deep learning networks are separated in depth from the more common single-hidden layer neural networks; that is, the number of node layers that the data must pass through in a multi-step pattern recognition process. Previous versions of neural networks like the first sensors are shallow, consisting of an input and an output layer, and at most one hidden layer in between. More than three layers (including input and output) qualify as "deep" 
learning. In deep learning networks, each node layer is trained on a different set of features based on the output of the previous layer. The further you go into the neural network, the more complex the features your nodes can recognize, as they collect and recombine features from the previous layer (Pathmind, 2020).

\section{Deep neural network}

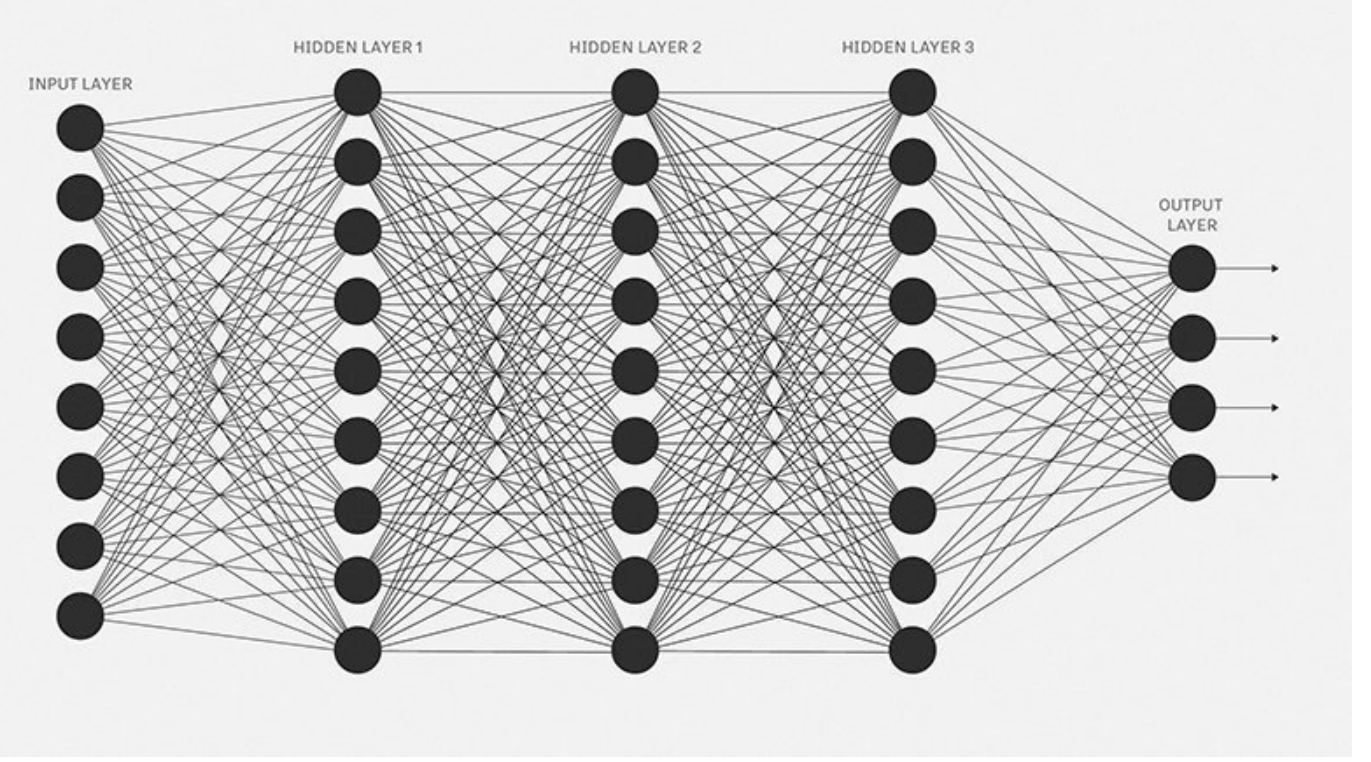

Figure 3. Deep neural network

Source: Willems, K. (2019).

\section{Application}

In this study, analyzes were made using the "TensorFlow", "Keras" and "Scikit-learn" libraries of the Phyton program. In addition to these, "Pandas", "Numpy", "Seaborn and" Matplotlib "libraries were also used. TensorFlow in Python is among the main platforms used in the research and development of Deep Learning. Creating deep learning models with TensorFlow is quite difficult. For this reason, Keras Python library is used to create a deep learning model on TensorFlow. Keras is a minimalist Python library for deep learning that can run on TensorFlow or Theano. Keras was developed to make the application of deep learning models for research and development as quick and easy as possible (Brownlee, 2019). Scikit-learn is a free library that provides many unsupervised and supervised learning algorithms in Python. It also has various algorithms such as the Scikit-learn support vector machine, random forests, and k-neighbors.

In that research, the possible market values of Borsa Istanbul firms are estimated by comparing the data of Forbes 2000 firms with the values of the firms in Borsa Istanbul. In the analysis, continuous value estimation from ANN types, one of the machine learning methods, was used. By this way, according to the sales, profit, assets and market value data of the Forbes 2000 company, the estimated market value was reached by entering the sales, profit and asset values of the companies entered from Borsa Istanbul.

Table 1. Number of layers and parameters created in the study

\begin{tabular}{|r|c|c|}
\hline Layer (type) & Output figure & Parameter \\
\hline 1. Layer (input) & $(256)$ & 1024 \\
\hline 2. Layer (hidden) & $(512)$ & 131584 \\
\hline 3. Layer (hidden) & $(256)$ & 131328 \\
\hline 4. Layer (hidden) & $(128)$ & 32896 \\
\hline 5. Layer (hidden) & $(64)$ & 8256 \\
\hline 6. Layer (output) & $(1)$ & 65 \\
\hline \multicolumn{2}{|l}{} \\
\hline \multicolumn{2}{|l}{ Total Parameter } & \\
\hline
\end{tabular}


The data of the Forbes 2000 companies for the years 2019-2018 are available from the website https://www.someka.net/excel-template/forbes-global-2000-list/, and the data of 389 companies in Borsa Istanbul are available to the Public Disclosure Platform. (KAP) has been accessed from the site https://www.kap.org.tr. The number of layers, outputs and parameters formed as a result of the Artificial Neural Network are given as in Table 1. Considering Table 1, since the number of data is high, it is seen that 1 Input layer has 4 Hidden layers and 1 Output layer. The high number of hidden layers in complex data provides better results. The summary of 2019 Forbes 2000 data, which was first entered into the Python program in the research, is shown in Table 2. At the same time, 2018 asset data were entered into the program in order to compare asset turnover ratios.

Table 2. 2018-2019 Forbes 2000 Data entered into the program (million \$)

\begin{tabular}{|c|l|l|c|c|c|c|c|c|}
\hline & Company & Country & Income & Profit & $\begin{array}{c}\text { Wealth } \\
2019\end{array}$ & $\begin{array}{c}\text { Wealth } \\
2018\end{array}$ & $\begin{array}{c}\text { Market } \\
\text { Value }\end{array}$ & Sectors \\
\hline 1 & ICBC & China & 175900 & 45200 & 4034500 & 4210900 & 305100 & Finance \\
\hline 2 & JPMorgan & USA & 132900 & 32700 & 2737200 & 2609800 & 368500 & Finance \\
\hline 3 & CCBank & China & 150300 & 38800 & 3382400 & 3631600 & 225000 & Finance \\
\hline$\ldots$ & $\ldots$ & $\ldots$ & $\ldots$ & $\ldots$ & $\ldots$ & $\ldots$ & $\ldots$ & $\ldots$ \\
\hline 1997 & Incyte & USA & 1900 & 109 & 2600 & 160 & 15800 & Medicine \\
\hline 1998 & F. G. Lease & Japan & 5600 & 209 & 23200 & 22900 & 1500 & Finance \\
\hline 1999 & JC Penney & USA & 12000 & -255 & 7900 & 8600 & 402 & Retail \\
\hline
\end{tabular}

Table 3. 2019 Forbes 2000 descriptive statistics

\begin{tabular}{|l|c|c|c|c|}
\cline { 2 - 5 } \multicolumn{1}{c|}{} & Incomes & Profit & Wealth & Market value \\
\hline Mean & 20579 & 1703 & 93349 & 28408 \\
\hline Std. & 35446 & 3724 & 284633 & 60025 \\
\hline Min & -9100 & -22400 & 1 & 9000 \\
\hline $25 \%$ & 4700 & 420 & 12000 & 6875 \\
\hline $50 \%$ & 10250 & 776 & 26050 & 13400 \\
\hline $75 \%$ & 20525 & 1700 & 60925 & 27500 \\
\hline Max & 514400 & 59400 & 4034500 & 961300 \\
\hline
\end{tabular}

Descriptive statistics of Forbes 2000 data are given in Table 3. The summary of Borsa İstanbul company data and ratio analysis included in the Python program in the 2. stage of the research is given in Table 4. Descriptive statistics of Borsa İstanbul data are shown in Table 5. (Except for ratio ratios, data are \$ million times.)

Table 4. 2019 and 2018 Borsa Istanbul data entered into the program (million \$)

\begin{tabular}{|c|l|c|c|c|c|c|c|c|c|l|}
\hline & Company & Income & Profit & Wealth & $\begin{array}{c}\text { Wealth } \\
2018\end{array}$ & $\begin{array}{c}\text { Return } \\
\text { on as- } \\
\text { sets }\end{array}$ & $\begin{array}{c}\text { Price } \\
\text { selling } \\
\text { ratio }\end{array}$ & $\begin{array}{c}\text { Asset } \\
\text { transfer } \\
\text { speed }\end{array}$ & $\begin{array}{c}\text { Share price } \\
\text { measure- } \\
\text { ment }\end{array}$ & Sectors \\
\hline 1 & ACSEL & 1.835 & 1.564 & 8,311 & 6,415 & 0,118 & 7,848 & 0,249 & 9,207 & Manufacture \\
\hline 2 & ADEL & -0.001 & $-0,002$ & 0,065 & 0,078 & $-0,038$ & -57228 & $-0,013$ & -21654 & Manufacture \\
\hline 3 & ADESE & 0,005 & $-0,000$ & 0,258 & 0,205 & $-0,000$ & 15352 & 0,22 & -910981 & Retail \\
\hline$\cdots$ & $\cdots$ & $\cdots$ & $\cdots$ & $\cdots$ & $\cdots$ & $\cdots$ & $\cdots$ & $\cdots$ & $\cdots$ & $\cdots$ \\
\hline 387 & YUNSA & 0.580 & 0,846 & 54,778 & 54,248 & 0,015 & 116,62 & 0,011 & 79,876 & Textile \\
\hline 388 & YYAPI & -6.764 & $-6,764$ & 101,179 & 92,962 & $-0,067$ & $-4,908$ & $-0,070$ & $-4,908$ & Construction \\
\hline 389 & ZOREN & $-0,123$ & $-0,027$ & 3,319 & 2,885 & $-0,008$ & $-6035,8$ & $-0,041$ & -27883 & Electric \\
\hline
\end{tabular}

Table 5. Descriptive statistics of Borsa İstanbul data (excluding Ratios $\mathrm{m} \$$ )

\begin{tabular}{|l|c|c|c|c|c|c|c|c|}
\cline { 2 - 9 } \multicolumn{1}{c|}{} & $\begin{array}{c}\text { Incomes } \\
2019\end{array}$ & $\begin{array}{c}\text { Profit } \\
2019\end{array}$ & $\begin{array}{c}\text { Wealth } \\
2019\end{array}$ & $\begin{array}{c}\text { Wealth } \\
2018\end{array}$ & $\begin{array}{c}\text { Return on } \\
\text { assets }\end{array}$ & $\begin{array}{c}\text { Price selling } \\
\text { ratio }\end{array}$ & $\begin{array}{c}\text { Asset transfer } \\
\text { speed }\end{array}$ & $\begin{array}{c}\text { Share price } \\
\text { measurement }\end{array}$ \\
\hline Mean & 7,11 & 4,780 & 171,0 & 141,7 & 0,027 & 35472 & 0,060 & 252572 \\
\hline Std. & 31,08 & 23,326 & 435,6 & 333,9 & 0,184 & 488113 & 0,166 & 3413035 \\
\hline Min & $-80,27$ & $-124,4$ & 0,025 & 0,018 & $-2,221$ & -57228 & -1130 & -13359684 \\
\hline $25 \%$ & 0,005 & $-0,001$ & 7,805 & 6,707 & $-0,001$ & 1,906 & 0,002 & $-1,271$ \\
\hline $50 \%$ & 0,861 & 0,591 & 37,29 & 30,67 & 0,030 & 12,690 & 0,048 & 14,328 \\
\hline $75 \%$ & 5,943 & 5,091 & 149,8 & 130,5 & 0,087 & 51,120 & 0,113 & 48,670 \\
\hline Max & 360,4 & 224,2 & 4562,5 & 3399,9 & 0,453 & 9145257 & 1,440 & 56491478 \\
\hline
\end{tabular}

The income, profit, asset and market values of the companies included in Forbes 2000 were entered into the program, and then the income, profit, assets of the Turkish company data were entered one by one and the market 
value was requested from the program. At the same time, ratio calculations were made in order to measure this uncertainty and Table 6 includes the estimated and actual values and ratio ratios of Borsa Istanbul companies.

Table 6. Ratios and estimated market values of Borsa İstanbul firms

\begin{tabular}{|c|c|c|c|c|c|c|}
\hline Name & $\begin{array}{l}\text { Real market } \\
\text { value }\end{array}$ & $\begin{array}{l}\text { Estimated mar- } \\
\text { ket value }\end{array}$ & $\begin{array}{c}\text { Asset } \\
\text { transfer } \\
\text { speed }\end{array}$ & $\begin{array}{c}\text { Return } \\
\text { on assets }\end{array}$ & $\begin{array}{c}\text { Share price-meas- } \\
\text { urement }\end{array}$ & Price selling ratio \\
\hline ACSEL & 14,4 & 100,74 & 0,25 & 0,19 & 9,21 & 7,85 \\
\hline ADEL & 53,4 & 82,96 & $-0,01$ & $-0,04$ & $-21654,06$ & $-57228,03$ \\
\hline ADESE & 76,4 & 82,87 & 0,02 & 0,00 & $-910981,96$ & 15352,76 \\
\hline AEFEES & 1916,8 & 82,94 & 0,08 & 0,03 & 8800,55 & 3262,74 \\
\hline AFYON & 216,8 & 10,48 & $-0,09$ & $-0,09$ & $-21,15$ & $-21,14$ \\
\hline AGHOL & 728,1 & 82,43 & 0,06 & 0,02 & 3560,03 & 1272,03 \\
\hline AGYO & 73 & 131,82 & 0,06 & 0,06 & 13,97 & 14,03 \\
\hline AKBNK & 4356,2 & 80,18 & 0,02 & 0,01 & 4842,63 & 3383,14 \\
\hline AKCNS & 369,6 & 196,48 & 0,04 & 0,04 & 25,19 & 25,08 \\
\hline AKENR & 206,1 & 230,50 & 0,08 & $-0,11$ & $-1,66$ & 2,45 \\
\hline AKFGY & 100,5 & 179,98 & 0,03 & 0,03 & 7,52 & 7,52 \\
\hline AKGRT & 321,9 & 755,36 & $-0,01$ & 0,11 & 3,89 & $-48,52$ \\
\hline AKGUV & 17,7 & 16,47 & $-0,18$ & $-0,21$ & $-4,78$ & $-4,77$ \\
\hline AKMGY & 193,9 & 249,58 & 0,31 & 0,32 & 14,15 & 14,28 \\
\hline AKSA & 336,8 & 83,24 & 0,07 & 0,07 & 7216,74 & 6791,17 \\
\hline AKSEN & 502,1 & 1420,06 & 0,44 & 0,08 & 6,56 & 1,39 \\
\hline AKSGY & 253 & 1023,45 & 0,08 & 0,09 & 2,74 & 3,02 \\
\hline AKSUE & 26,5 & 83,19 & 0,03 & 0,03 & 81,51 & 80,78 \\
\hline ALARK & 343,5 & 1019,98 & 0,30 & 0,27 & 4,48 & 4,32 \\
\hline ALBRK & 322,6 & 79,29 & 0,00 & 0,00 & 30261,71 & 12047,66 \\
\hline ALCAR & 119,7 & 92,19 & 0,02 & 0,03 & 43,44 & 50,66 \\
\hline ALCTL & 106,3 & 10,57 & 0,00 & $-0,04$ & $-21,84$ & $-751,65$ \\
\hline ALGYO & 159,8 & 878,19 & 0,29 & 0,25 & 2,44 & 2,40 \\
\hline ALKA & 82,9 & 171,38 & 0,19 & 0,19 & 11,09 & 11,28 \\
\hline ALKIM & 266,6 & 366,94 & 0,27 & 0,25 & 11,30 & 11,54 \\
\hline ALYAG & 18,7 & 95,58 & 0,08 & 0,10 & 14,94 & 15,16 \\
\hline ANACM & 571,5 & 83,54 & 0,10 & 0,07 & 6449,68 & 5283,74 \\
\hline ANELE & 76,3 & 59,90 & 0,02 & 0,00 & 142,21 & 14,79 \\
\hline ANHYT & 516,8 & 675,91 & 0,00 & 0,02 & 6,45 & $-12247,03$ \\
\hline ANSGR & 461,1 & 861,37 & 0,00 & 0,06 & 4,83 & 808,45 \\
\hline ARCLK & 1944,4 & 81,75 & 0,05 & 0,03 & 12139,42 & 7642,45 \\
\hline ARDYZ & 104,6 & 136,14 & 0,34 & 0,30 & 23,57 & 23,54 \\
\hline ARENA & 77,4 & 115,22 & 0,07 & 0,03 & 15,25 & 6,03 \\
\hline ARMDA & 80,8 & 79,22 & 0,04 & 0,01 & 37,15 & 11,15 \\
\hline ARSAN & 63,4 & 195,78 & 0,11 & 0,09 & 6,32 & 5,63 \\
\hline ARTI & 2,9 & 10,94 & $-1,13$ & $-2,22$ & $-0,61$ & $-0,61$ \\
\hline ASELS & 5278,3 & 88,43 & 0,16 & 0,13 & 9367,42 & 8841,71 \\
\hline ASUZU & 168 & 86,53 & 0,01 & 0,01 & 45,88 & 51,39 \\
\hline ATAGY & 12 & 60,32 & $-0,08$ & $-0,08$ & $-9,49$ & $-9,49$ \\
\hline ATEKS & 48,4 & 82,22 & 0,04 & 0,02 & 23,90 & 10,24 \\
\hline ATLAS & 7,5 & 103,66 & 0,25 & 0,23 & 4,12 & 4,11 \\
\hline ATSYH & 2,3 & 81,02 & 0,04 & 0,00 & 18619,05 & 23,13 \\
\hline AVGYO & 26,1 & 150,77 & 0,18 & 0,16 & 4,58 & 4,40 \\
\hline AVHOL & 7,2 & 85,93 & 0,06 & 0,06 & 14,39 & 14,51 \\
\hline AVISA & 389,7 & 357,26 & 0,00 & 0,01 & 8,49 & 170,16 \\
\hline AVOD & 44,1 & 112,94 & 0,10 & 0,06 & 15,73 & 11,95 \\
\hline AVTUR & 13,1 & 95,15 & 0,07 & 0,07 & 10,43 & 10,49 \\
\hline AYCES & 145,8 & 98,74 & 0,30 & 0,01 & 313,36 & 12,40 \\
\hline AYEN & 258,7 & 88,48 & 0,03 & 0,01 & 75,58 & 16,78 \\
\hline AYES & 8,8 & 82,81 & 0,02 & 0,02 & 25,49 & 25,83 \\
\hline AYGAZ & 497,8 & 83,17 & 0,07 & 0,06 & 10830,56 & 8189,47 \\
\hline
\end{tabular}

Copyright (C) 2021 by IJSSER

ISSN: 2149-5939 


\begin{tabular}{|c|c|c|c|c|c|c|}
\hline BAGFS & 108,5 & 192,82 & 0,06 & 0,05 & 7,89 & 7,88 \\
\hline BAKAB & 46,6 & 94,13 & 0,04 & 0,03 & 22,14 & 15,02 \\
\hline BALAT & 3,6 & 77,72 & $-0,04$ & $-0,04$ & $-22,47$ & $-22,45$ \\
\hline BANVT & 355,3 & 344,45 & 0,20 & 0,05 & 19,12 & 5,83 \\
\hline BASCM & 81,5 & 56,71 & 0,00 & 0,00 & 254,51 & 304,09 \\
\hline BERA & 264,8 & 200,52 & 0,02 & 0,03 & 16,29 & 23,92 \\
\hline BEYAZ & 55,2 & 104,01 & 0,06 & 0,05 & 22,70 & 24,30 \\
\hline BFREN & 137,2 & 137,71 & 0,25 & 0,25 & 29,82 & 30,45 \\
\hline BIZIM & 170 & 107,21 & 0,03 & 0,03 & 30,58 & 36,48 \\
\hline BİMAS & 6028,8 & 84,35 & 0,11 & 0,08 & 29285,68 & 26483,63 \\
\hline BJKAS & 111,5 & 52,07 & $-0,45$ & $-0,41$ & $-1,39$ & $-1,39$ \\
\hline BLCYT & 51,1 & 235,97 & 0,25 & 0,25 & 4,03 & 4,04 \\
\hline BMELK & 13,9 & 86,31 & 0,34 & 0,01 & 89,90 & 4,06 \\
\hline BNTAS & 35,6 & 110,10 & 0,10 & 0,09 & 14,43 & 14,43 \\
\hline BOSSA & 85,6 & 197,58 & 0,09 & 0,07 & 7,89 & 6,79 \\
\hline BRISA & 546,8 & 204,18 & 0,00 & 0,02 & 28,54 & $-141,20$ \\
\hline BRKO & 8,2 & 48,49 & $-0,08$ & $-0,08$ & $-4,40$ & $-4,32$ \\
\hline BRKSN & 9,4 & 74,98 & $-0,03$ & $-0,02$ & $-33,37$ & $-29,97$ \\
\hline BRMEN & 3,8 & 52,19 & $-0,11$ & $-0,13$ & $-2,12$ & $-2,21$ \\
\hline BRSAN & 243,1 & 154,43 & 0,04 & 0,01 & 22,01 & 5,15 \\
\hline BRYAT & 322,9 & 208,59 & 0,15 & 0,03 & 28,43 & 5,90 \\
\hline BSKOE & 93,2 & 25,66 & $-0,15$ & $-0,15$ & $-2,79$ & $-2,86$ \\
\hline BTCIM & 88,4 & 27,06 & $-0,10$ & $-0,10$ & $-2,26$ & $-2,23$ \\
\hline BUCIM & 115,7 & 113,58 & 0,03 & 0,04 & 20,31 & 22,85 \\
\hline BURCE & 13,5 & 80,93 & 0,32 & $-0,02$ & $-27,94$ & 2,16 \\
\hline BURVA & 30,6 & 81,26 & 0,08 & $-0,01$ & $-1228,90$ & 141,79 \\
\hline CASA & 14,6 & 96,30 & $-0,11$ & 0,13 & 7,00 & $-8,61$ \\
\hline CCOLA & 1652,1 & 83,64 & 0,08 & 0,06 & 10432,51 & 7892,93 \\
\hline CELHA & 25,9 & 47,80 & 0,00 & $-0,04$ & $-14,75$ & $-153,67$ \\
\hline CEMAS & 109,8 & 20,15 & 0,02 & $-0,06$ & $-31,02$ & 95,47 \\
\hline CEMTS & 121,4 & 187,23 & 0,10 & 0,10 & 12,80 & 13,22 \\
\hline CEOEM & 26,1 & 81,17 & 0,02 & 0,01 & 136,52 & 141,99 \\
\hline CIMSA & 205,5 & 20,48 & 0,01 & $-0,01$ & $-38,21$ & 35,66 \\
\hline CLEBI & 269,1 & 451,92 & 0,13 & 0,09 & 8,19 & 6,72 \\
\hline CMBTN & 15,2 & 71,16 & $-0,03$ & $-0,02$ & $-33,72$ & $-28,58$ \\
\hline CMENT & 291,4 & 82,58 & $-0,06$ & $-0,06$ & $-16696,49$ & $-16173,64$ \\
\hline COSMO & 2,4 & 90,76 & 0,17 & 0,17 & 3,26 & 3,42 \\
\hline CRDFA & 52,8 & 116,42 & 0,05 & 0,05 & 13,54 & 13,57 \\
\hline CRFSA & 736,3 & 37,84 & $-0,11$ & $-0,09$ & $-12,77$ & $-12,33$ \\
\hline CUSAN & 38,4 & 94,78 & 0,02 & 0,04 & 20,17 & 31,45 \\
\hline DAGHL & 11,5 & 84,58 & 0,09 & 0,08 & 42,95 & 43,26 \\
\hline DAGI & 39,9 & 86,75 & 0,03 & 0,03 & 45,05 & 47,81 \\
\hline DARDL & 89,3 & 88,59 & 0,04 & 0,03 & 99,82 & 94,91 \\
\hline DENCM & 17,6 & 62,87 & $-0,05$ & $-0,05$ & $-17,52$ & $-15,61$ \\
\hline DENGE & 12,2 & 8,60 & $-0,67$ & $-1,03$ & $-1,35$ & $-1,35$ \\
\hline DERAS & 26,2 & 86,85 & 0,04 & 0,04 & 40,70 & 43,96 \\
\hline DERIM & 26,3 & 50,50 & $-0,02$ & $-0,02$ & $-24,74$ & $-23,29$ \\
\hline DESA & 35,5 & 109,00 & 0,05 & 0,05 & 11,58 & 12,49 \\
\hline DESPC & 25 & 105,40 & 0,09 & 0,08 & 11,93 & 11,95 \\
\hline DEVA & 559,5 & 634,61 & 0,15 & 0,13 & 11,64 & 11,61 \\
\hline DGATE & 39,2 & 125,89 & 0,09 & 0,09 & 9,91 & 9,91 \\
\hline DGGYO & 270,9 & 211,09 & 0,05 & 0,05 & 16,96 & 16,96 \\
\hline DGKLB & 95,7 & 11,12 & $-0,08$ & $-0,08$ & $-8,73$ & $-10,68$ \\
\hline DIRIT & 1,9 & 64,22 & $-0,38$ & $-0,52$ & $-1,68$ & $-1,68$ \\
\hline DITAS & 36,1 & 80,98 & 0,01 & 0,01 & 198,14 & 266,51 \\
\hline DMSAS & 45,5 & 108,06 & 0,05 & 0,05 & 16,68 & 16,37 \\
\hline DOAS & 483,3 & 82,67 & 0,01 & 0,02 & 37463,16 & 43458,95 \\
\hline DOBUR & 46 & 73,09 & $-0,06$ & $-0,04$ & $-110,30$ & $-87,82$ \\
\hline
\end{tabular}

Copyright (C) 2021 by IJSSER

ISSN: 2149-5939 


\begin{tabular}{|c|c|c|c|c|c|c|}
\hline DOGUB & 13,1 & 74,79 & 0,05 & $-0,11$ & $-28,79$ & 66,89 \\
\hline DOHOL & 838,8 & 83,23 & 0,06 & 0,05 & 8417,24 & 7273,46 \\
\hline DOKTA & 407,3 & 248,34 & 0,08 & 0,07 & 24,69 & 22,01 \\
\hline DURDO & 39,2 & 94,35 & 0,03 & 0,04 & 23,04 & 29,08 \\
\hline DYOBY & 93,8 & 94,38 & 0,05 & 0,02 & 27,62 & 9,84 \\
\hline DZGYO & 39,3 & 76,04 & 0,02 & 0,02 & 17,52 & 17,56 \\
\hline ECILC & 622 & 83,02 & 0,10 & 0,03 & 22742,86 & 7649,78 \\
\hline ECZYT & 223 & 216,40 & 0,13 & 0,03 & 18,27 & 4,47 \\
\hline EDIP & 21,9 & 108,99 & 0,04 & 0,03 & 4,51 & 4,50 \\
\hline EGCEY & 3,3 & 79,61 & 0,00 & $-0,02$ & $-46,85$ & $-47199,52$ \\
\hline EGCYH & 4,7 & 83,99 & 0,05 & 0,12 & 44,80 & 44,71 \\
\hline EGGUB & 122,1 & 163,23 & 0,10 & 0,08 & 16,03 & 16,14 \\
\hline EGPRO & 170,4 & 114,19 & 0,03 & 0,03 & 27,41 & 28,54 \\
\hline EGSER & 55,6 & 120,42 & 0,06 & 0,05 & 13,37 & 12,09 \\
\hline EKGYO & 1195,8 & 80,22 & 0,00 & 0,00 & 9145257,07 & 9145257,07 \\
\hline EKIZ & 10,7 & 86,27 & 0,07 & 0,07 & 20,52 & 20,74 \\
\hline EMKEL & 9,7 & 76,42 & 0,06 & $-0,01$ & $-50,54$ & 4,56 \\
\hline EMNIS & 3,3 & 46,79 & $-0,24$ & $-0,24$ & $-1,54$ & $-1,53$ \\
\hline ENJSA & 1481,5 & 82,86 & 0,04 & 0,04 & 8528,19 & 8744,84 \\
\hline ENKAI & 4870,8 & 89,02 & 0,15 & 0,08 & 7491,19 & 4284,85 \\
\hline EPLAS & 48,4 & 82,25 & 0,01 & 0,02 & 111,38 & 134,65 \\
\hline ERBOS & 62,2 & 206,73 & 0,12 & 0,11 & 5,65 & 5,70 \\
\hline EREGL & 4385,3 & 88,38 & 0,16 & 0,07 & 7467,37 & 3790,78 \\
\hline ERSU & 22,2 & 84,23 & $-0,01$ & 0,05 & 46,38 & $-281,81$ \\
\hline ESCOM & 26,4 & 40,54 & $-0,21$ & $-0,24$ & $-10,66$ & $-10,66$ \\
\hline ETILR & 4,7 & 81,10 & $-0,01$ & $-0,02$ & $-137,81$ & $-135,77$ \\
\hline ETYAT & 5 & 99,56 & 0,30 & 0,27 & 3,57 & 3,58 \\
\hline EUHOL & 15,1 & 83,27 & 0,03 & 0,02 & 44,34 & 44,68 \\
\hline EUKYO & 5,6 & 99,76 & 0,28 & 0,25 & 3,90 & 3,91 \\
\hline EUYO & 5 & 99,30 & 0,29 & 0,27 & 3,63 & 3,64 \\
\hline FENER & 177,4 & 18,98 & $-0,10$ & $-0,08$ & $-6,97$ & $-6,90$ \\
\hline FLAP & 18,8 & 94,28 & 0,11 & 0,06 & 17,59 & 10,64 \\
\hline FMIZP & 112,1 & 149,28 & 0,48 & 0,45 & 21,15 & 21,14 \\
\hline FONET & 60,6 & 111,00 & 0,19 & 0,18 & 24,61 & 25,19 \\
\hline FORMT & 32,1 & 24,58 & $-0,12$ & $-0,12$ & $-10,41$ & $-10,53$ \\
\hline FRIGO & 15,7 & 103,98 & 0,14 & 0,13 & 8,17 & 8,51 \\
\hline FROTO & 3732,1 & 86,01 & 0,14 & 0,12 & 11332,57 & 10800,51 \\
\hline GARFA & 57,8 & 82,84 & 0,01 & 0,01 & 14072,76 & 14054,93 \\
\hline GEDIK & 159,4 & 181,02 & 0,07 & 0,06 & 14,52 & 14,66 \\
\hline GEDZA & 18,3 & 107,32 & 0,13 & 0,12 & 8,38 & 8,44 \\
\hline GENTS & 62,6 & 92,39 & 0,03 & 0,03 & 32,61 & 33,23 \\
\hline GEREL & 59,1 & 9,15 & $-0,17$ & $-0,14$ & $-9,83$ & $-7,64$ \\
\hline GLRYH & 63,8 & 99,71 & 0,05 & 0,04 & 31,59 & 32,26 \\
\hline GLYHO & 193,7 & 26,18 & $-0,03$ & $-0,02$ & $-7,31$ & $-7,07$ \\
\hline GOLTS & 49,2 & 126,60 & 0,04 & 0,04 & 7,92 & 7,90 \\
\hline GOODY & 210,5 & 616,03 & 0,17 & 0,17 & 4,65 & 4,65 \\
\hline GOZDE & 263,6 & 29,71 & 0,00 & 0,00 & $-176,11$ & $-176,11$ \\
\hline GSDDE & 29,6 & 83,00 & 0,04 & $-0,02$ & $-30656,22$ & 17770,15 \\
\hline GSDHO & 135,7 & 83,21 & 0,10 & 0,08 & 4915,65 & 3885,61 \\
\hline GSRAY & 209,3 & 11,39 & $-0,04$ & $-0,03$ & $-26,66$ & $-26,46$ \\
\hline GUBRF & 1295,4 & 20,54 & $-0,07$ & $-0,02$ & $-78,14$ & $-25,15$ \\
\hline GUSGR & 326,5 & 246,36 & 0,00 & 0,04 & 14,28 & 30700,64 \\
\hline GYHOL & 95,5 & 255,71 & 0,52 & 0,41 & 6,78 & 6,78 \\
\hline HALKB,THL & 2126,4 & 65,40 & 0,01 & 0,00 & 7354,54 & 3883,10 \\
\hline HALKS & 189,1 & 493,45 & 0,00 & 0,10 & 3,73 & 322,64 \\
\hline HATEK & 28,3 & 162,34 & 0,28 & 0,07 & 5,88 & 1,54 \\
\hline HDFGS & 26,8 & 144,37 & 0,26 & 0,23 & 5,22 & 5,22 \\
\hline HEKTS & 450,9 & 358,73 & 0,15 & 0,12 & 18,43 & 18,51 \\
\hline
\end{tabular}

Copyright (C) 2021 by IJSSER

ISSN: 2149-5939 


\begin{tabular}{|c|c|c|c|c|c|c|}
\hline HLGYO & 301,5 & 677,37 & 0,11 & 0,11 & 5,58 & 5,58 \\
\hline HUBVC & 86,1 & 94,50 & 0,14 & 0,13 & 75,37 & 75,38 \\
\hline HURGZ & 120,7 & 13,22 & 0,02 & $-0,06$ & $-14,52$ & 51,03 \\
\hline ICB & 674,1 & 80,99 & 0,01 & 0,00 & 56491478,87 & 24645,58 \\
\hline IDEAS & 5,3 & 81,24 & $-0,01$ & 0,00 & 939,52 & $-154,65$ \\
\hline IDGYO & 5,9 & 81,79 & 0,02 & 0,02 & 128,31 & 136,38 \\
\hline IEYHO & 75,2 & 13,69 & 0,06 & $-0,06$ & $-14,94$ & 14,25 \\
\hline IHEVA & 109,8 & 166,80 & 0,14 & 0,09 & 15,24 & 12,14 \\
\hline IHGZT & 68 & 114,32 & 0,05 & 0,06 & 19,97 & 24,32 \\
\hline IHLAS & 96,7 & 46,73 & 0,00 & 0,00 & 109,35 & 89,60 \\
\hline IHLGM & 160 & 122,20 & 0,05 & 0,03 & 22,39 & 22,94 \\
\hline IHYAY & 32,9 & 89,84 & 0,02 & 0,03 & 17,49 & 27,34 \\
\hline INDES & 86 & 220,07 & 0,05 & 0,05 & 5,01 & 5,02 \\
\hline INFO & 25,1 & 83,48 & 0,03 & 0,02 & 31,97 & 31,09 \\
\hline INTEM & 35 & 62,30 & 0,00 & 0,00 & $-719,31$ & $-719,31$ \\
\hline IPEKE & 528 & 86,63 & 0,31 & 0,26 & 1773,48 & 1769,49 \\
\hline ISATR,ISBTR & 42,8 & 76,03 & 0,02 & 0,01 & 41,97 & 27,56 \\
\hline ISBIR & 113,8 & 134,13 & 0,05 & 0,05 & 16,94 & 17,99 \\
\hline ISDMR & 3037,8 & 86,68 & 0,19 & 0,10 & 8385,76 & 4549,48 \\
\hline ISFIN & 387 & 82,03 & 0,01 & 0,00 & 29147468,35 & 18667,16 \\
\hline ISGSY & 81,9 & 75,83 & 0,01 & 0,01 & 280,69 & 269,50 \\
\hline ISGYO & 282,2 & 535,44 & 0,06 & 0,05 & 5,65 & 5,54 \\
\hline ISMEN & 337,7 & 746,12 & 0,07 & 0,06 & 4,77 & 4,72 \\
\hline ISYAT & 59 & 212,90 & 0,25 & 0,23 & 5,46 & 5,46 \\
\hline ITTFH & 43,7 & 82,78 & 0,02 & 0,00 & $-3823750,00$ & 7603,89 \\
\hline IZFAS & 7,1 & 80,29 & 0,01 & 0,01 & 82,76 & 148,82 \\
\hline IZMDC & 888,9 & 123,62 & 0,00 & $-0,13$ & $-10,00$ & 507,98 \\
\hline IZTAR & 11,2 & 76,82 & 0,26 & $-0,04$ & $-23,77$ & 4,49 \\
\hline JANTS & 120 & 242,46 & 0,22 & 0,19 & 9,07 & 8,69 \\
\hline KAPLM & 28,4 & 68,31 & $-0,03$ & $-0,02$ & $-51,47$ & $-40,52$ \\
\hline KAREL & 134,6 & 288,57 & 0,11 & 0,10 & 7,10 & 7,40 \\
\hline KARSN & 449,8 & 84,72 & 0,02 & 0,01 & 131,81 & 59,32 \\
\hline KARTN & 202,5 & 242,00 & 0,16 & 0,15 & 14,80 & 14,97 \\
\hline KATMR & 51,1 & 26,10 & $-0,02$ & $-0,02$ & $-22,46$ & $-22,88$ \\
\hline KCHOL & 6332,5 & 90,64 & 0,04 & 0,04 & 6363,21 & 6244,84 \\
\hline KENT & 3960,1 & 96,55 & 0,03 & 0,03 & 955,16 & 1119,69 \\
\hline KERVN & 40,3 & 27,22 & $-0,11$ & $-0,17$ & $-1,45$ & $-2,15$ \\
\hline KERVT & 469,7 & 329,92 & 0,06 & 0,06 & 17,59 & 15,16 \\
\hline KFEIN & 52,4 & 126,52 & 0,16 & 0,14 & 13,94 & 14,03 \\
\hline KLGYO & 106,6 & 81,76 & $-0,25$ & $-0,32$ & $-1416,93$ & $-1416,74$ \\
\hline KLMSN & 90,3 & 290,35 & 0,11 & 0,09 & 4,76 & 4,58 \\
\hline KLN & 5010,5 & 81,90 & 0,03 & 0,02 & 66678,76 & 61351,88 \\
\hline KNFRT & 48,7 & 122,44 & 0,07 & 0,08 & 12,24 & 15,57 \\
\hline KONYA & 208,5 & 63,02 & 0,00 & 0,00 & 2005,85 & $-11621,09$ \\
\hline KORDS & 340,9 & 726,30 & 0,10 & 0,05 & 5,36 & 3,27 \\
\hline KOZAA & 729,4 & 86,64 & 0,31 & 0,26 & 2447,63 & 2445,90 \\
\hline KOZAL & 1801,9 & 86,66 & 0,33 & 0,28 & 6066,52 & 6054,67 \\
\hline KPHOL & 2,2 & 82,91 & 0,04 & 0,04 & 42,56 & 42,56 \\
\hline KRDMA & 150,5 & 143,20 & 0,01 & 0,01 & 11,10 & 13,94 \\
\hline KRGYO & 28,8 & 108,11 & 0,12 & 0,11 & 12,77 & 12,79 \\
\hline KRONT & 55,7 & 103,13 & 0,15 & 0,14 & 29,81 & 33,11 \\
\hline KRSTL & 28,8 & 77,90 & 0,00 & 0,00 & 248,57 & 228,55 \\
\hline KRTEK & 25 & 73,71 & 0,01 & 0,01 & 48,60 & 46,78 \\
\hline KSTUR & 34 & 110,50 & 0,25 & 0,26 & 14,41 & 14,41 \\
\hline KUTPO & 231,5 & 154,48 & 0,08 & 0,08 & 33,99 & 36,58 \\
\hline KUYAS & 22,6 & 12,27 & $-0,11$ & $-0,13$ & $-5,45$ & $-5,72$ \\
\hline LIDFA & 42,5 & 82,93 & 0,01 & 0,00 & 14048611,11 & 17891,26 \\
\hline LINK & 19,6 & 96,44 & 0,20 & 0,18 & 15,66 & 15,83 \\
\hline
\end{tabular}




\begin{tabular}{|c|c|c|c|c|c|c|}
\hline LKMNH & 32,3 & 86,57 & 0,03 & 0,02 & 23,24 & 18,46 \\
\hline LOGO & 320,7 & 248,98 & 0,14 & 0,11 & 22,11 & 20,48 \\
\hline LUKSK & 31,8 & 99,28 & 0,06 & 0,06 & 18,50 & 20,73 \\
\hline MAALT & 83,1 & 115,46 & 0,11 & 0,10 & 28,77 & 28,79 \\
\hline MAKTK & 67 & 112,24 & 0,30 & 0,06 & 37,64 & 8,70 \\
\hline MARKA & 8,1 & 89,52 & 0,14 & 0,14 & 12,14 & 12,13 \\
\hline MARTI & 21 & 26,31 & $-0,02$ & $-0,10$ & $-0,98$ & $-4,58$ \\
\hline MAVI & 323,7 & 83,09 & 0,09 & 0,06 & 17852,15 & 13708,68 \\
\hline MEGAP & 9,5 & 93,41 & 0,09 & 0,06 & 8,38 & 8,40 \\
\hline MEPET & 40,2 & 61,71 & 0,01 & $-0,01$ & $-101,99$ & 50,42 \\
\hline MERIT & 8,3 & 94,71 & 0,11 & 0,14 & 6,85 & 9,05 \\
\hline MERKO & 13,1 & 36,13 & $-0,18$ & $-0,18$ & $-4,94$ & $-4,94$ \\
\hline METRO & 77,8 & 334,67 & 0,13 & 0,12 & 3,50 & 3,51 \\
\hline METUR & 6,2 & 79,36 & 0,00 & 0,00 & 12903,11 & $-12118,92$ \\
\hline MGROS & 1104,7 & 81,20 & $-0,02$ & 0,00 & $-13359684,96$ & $-21052,22$ \\
\hline MIPAZ & 114,2 & 257,42 & 0,35 & 0,28 & 7,95 & 7,95 \\
\hline MMCAS & 3,4 & 80,76 & $-0,02$ & $-0,01$ & $-93,95$ & $-95,05$ \\
\hline MNDRS & 111,5 & 17,98 & $-0,01$ & $-0,01$ & $-37,48$ & $-36,92$ \\
\hline MPARK & 607,4 & 82,71 & 0,01 & 0,01 & 64244,85 & 81850,57 \\
\hline MRGYO & 36,7 & 109,26 & 0,09 & 0,03 & 11,60 & 3,49 \\
\hline MRSHL & 120,8 & 113,78 & 0,07 & 0,06 & 38,33 & 38,79 \\
\hline MSGYO & 46,7 & 151,07 & 0,14 & 0,14 & 7,96 & 7,97 \\
\hline MTRYO & 14,6 & 109,05 & 0,27 & 0,24 & 6,40 & 6,40 \\
\hline MZHDL & 26,1 & 83,53 & 0,02 & 0,02 & 48,89 & 57,39 \\
\hline NATEN & 122,6 & 210,66 & 0,16 & 0,12 & 10,76 & 11,31 \\
\hline NETAS & 140,5 & 22,04 & $-0,04$ & $-0,07$ & $-5,62$ & $-10,20$ \\
\hline NIBAS & 110,2 & 97,22 & 0,47 & 0,03 & 186,29 & 14,80 \\
\hline NTHOL & 194,7 & 215,46 & 0,09 & 0,01 & 13,23 & 1,95 \\
\hline NUGYO & 190,8 & 37,13 & $-0,16$ & $-0,17$ & $-3,91$ & $-4,02$ \\
\hline NUHCM & 350,4 & 366,86 & 0,08 & 0,09 & 12,97 & 13,28 \\
\hline ODAS & 235,1 & 20,00 & $-0,09$ & $-0,06$ & $-7,50$ & $-4,68$ \\
\hline OLMIP & 190,4 & 13,24 & $-0,11$ & $-0,12$ & $-12,30$ & $-11,95$ \\
\hline ORGE & 50 & 172,19 & 0,18 & 0,15 & 6,57 & 6,61 \\
\hline ORMA & 29,1 & 16,06 & $-0,01$ & $-0,02$ & $-8,75$ & $-15,27$ \\
\hline OSTIM & 32,7 & 80,90 & 0,01 & 0,01 & 47,19 & 49,14 \\
\hline OTKAR & 547,2 & 83,45 & 0,01 & 0,13 & 9260,28 & 101725,93 \\
\hline OYA & 19,5 & 199,18 & 0,08 & 0,07 & 1,66 & 1,67 \\
\hline OYAKC & 1427,8 & 130,15 & 0,06 & 0,10 & 314,62 & 411,77 \\
\hline OYLUM & 12,8 & 83,01 & 0,03 & 0,03 & 42,64 & 44,07 \\
\hline OZBAL & 24,4 & 49,91 & $-0,04$ & $-0,09$ & $-12,55$ & $-31,88$ \\
\hline OZGYO & 82 & 29,66 & $-0,02$ & $-0,03$ & $-37,83$ & $-38,22$ \\
\hline OZKGY & 224,3 & 1202,37 & 0,14 & 0,12 & 2,27 & 2,21 \\
\hline OZRDN & 7 & 65,50 & $-0,09$ & $-0,08$ & $-7,48$ & $-7,19$ \\
\hline PAGYO & 69,5 & 182,90 & 0,06 & 0,06 & 6,61 & 6,61 \\
\hline PAPIL & 79,6 & 97,16 & 0,11 & 0,09 & 57,24 & 57,38 \\
\hline PARSN & 209 & 83,14 & 0,12 & 0,01 & 129,37 & 6,03 \\
\hline PEGYO & 19 & 57,21 & $-0,06$ & $-0,06$ & $-13,78$ & $-13,75$ \\
\hline PEKGY & 92,7 & 53,57 & 0,00 & 0,00 & $-503,08$ & $-514,07$ \\
\hline PENGD & 33,5 & 68,85 & 0,51 & $-0,02$ & $-23,88$ & 1,44 \\
\hline PETKM & 1283,1 & 83,18 & 0,05 & 0,05 & 9989,63 & 10693,32 \\
\hline PETUN & 116 & 193,66 & 0,19 & 0,05 & 14,94 & 4,68 \\
\hline PGSUS & 769,8 & 2425,18 & 0,11 & 0,06 & 3,43 & 2,36 \\
\hline PINSU & 35,9 & 9,76 & $-0,04$ & $-0,10$ & $-6,52$ & $-16,68$ \\
\hline PKART & 47,3 & 97,25 & 0,09 & 0,08 & 33,52 & 33,80 \\
\hline PKENT & 41,8 & 107,43 & 0,18 & 0,21 & 19,10 & 20,49 \\
\hline PNSUT & 118,9 & 129,75 & 0,10 & 0,02 & 20,40 & 5,03 \\
\hline POLHO & 327,1 & 152,71 & 0,02 & 0,03 & 30,77 & 34,72 \\
\hline POLTK & 74,8 & 88,45 & 0,10 & 0,10 & 111,16 & 108,62 \\
\hline
\end{tabular}

Copyright (C) 2021 by IJSSER

ISSN: 2149-5939 


\begin{tabular}{|c|c|c|c|c|c|c|}
\hline PRKAB & 178,4 & 121,16 & 0,04 & 0,04 & 29,51 & 33,18 \\
\hline PRKME & 85,5 & 65,68 & 0,01 & 0,01 & 125,44 & 80,65 \\
\hline PRZMA & 14,4 & 81,43 & 0,02 & 0,02 & 83,45 & 92,97 \\
\hline PSDTC & 11,1 & 70,79 & 0,01 & 0,01 & 7,23 & 7,25 \\
\hline QNBFF & 21143,1 & 82,95 & 0,03 & 0,03 & 2605232,05 & 2617918,28 \\
\hline RALYH & 52,8 & 80,45 & 0,01 & 0,01 & 1131,66 & 1397,69 \\
\hline RAYSG & 138,3 & 149,26 & 0,00 & 0,05 & 12,53 & $-3653,55$ \\
\hline RHEAG & 12,7 & 80,58 & 0,02 & 0,02 & 148,79 & 148,79 \\
\hline RODRG & 2,6 & 83,31 & 0,08 & 0,05 & 13,37 & 8,09 \\
\hline ROYAL & 18,4 & 13,63 & $-0,14$ & $-0,15$ & $-1,25$ & $-1,37$ \\
\hline RTALB & 223,8 & 88,68 & 0,05 & 0,05 & 291,22 & 303,28 \\
\hline RYGYO & 192,5 & 1182,30 & 0,22 & 0,20 & 2,09 & 2,09 \\
\hline RYSAS & 101,7 & 57,31 & 0,00 & 0,00 & 134,10 & 117,87 \\
\hline SAFKR & 22,1 & 90,74 & 0,06 & 0,06 & 24,27 & 24,76 \\
\hline SAHOL & 2705,2 & 84,62 & 0,02 & 0,02 & 1883,85 & 1724,56 \\
\hline SALIX & 7,3 & 81,99 & $-0,02$ & $-0,06$ & $-171,55$ & $-495,08$ \\
\hline SAMAT & 15,9 & 43,41 & $-0,14$ & $-0,16$ & $-7,01$ & $-7,01$ \\
\hline SANEL & 10,1 & 86,39 & 0,07 & 0,07 & 18,97 & 19,22 \\
\hline SANFM & 10,7 & 86,41 & 0,03 & 0,04 & 17,47 & 23,56 \\
\hline SANKO & 110 & 152,66 & 0,10 & 0,10 & 17,56 & 17,55 \\
\hline SARKY & 208,3 & 198,73 & 0,05 & 0,04 & 13,94 & 13,43 \\
\hline SASA & 1321,7 & 82,64 & 0,18 & 0,00 & 624037,06 & 7389,84 \\
\hline SAYAS & 14,8 & 78,01 & $-0,01$ & $-0,01$ & $-170,24$ & $-190,28$ \\
\hline SEKFK & 32,3 & 82,95 & $-0,01$ & $-0,01$ & $-42099,67$ & $-28237,58$ \\
\hline SEKUR & 14,4 & 84,47 & 0,06 & 0,01 & 37,58 & 8,20 \\
\hline SELEC & 756,4 & 1273,74 & 0,09 & 0,08 & 6,48 & 6,59 \\
\hline SELGD & 4,6 & 89,29 & 0,27 & 0,10 & 8,42 & 3,58 \\
\hline SERVE & 10,7 & 63,43 & $-0,94$ & $-1,53$ & $-8,65$ & $-8,64$ \\
\hline SEYKM & 20,4 & 90,57 & 0,15 & 0,13 & 25,26 & 25,40 \\
\hline SILVR & 27,5 & 58,49 & $-0,04$ & $-0,03$ & $-25,68$ & $-23,02$ \\
\hline SISE & 2006,2 & 85,79 & 0,11 & 0,07 & 4420,55 & 3348,08 \\
\hline SKTAS & 32,1 & 28,36 & $-0,01$ & $-0,02$ & $-13,29$ & $-47,98$ \\
\hline SMART & 38,1 & 96,41 & 0,13 & 0,11 & 28,98 & 29,51 \\
\hline SNGYO & 183,2 & 398,85 & 0,04 & 0,04 & 5,04 & 5,12 \\
\hline SNKRN & 3,1 & 73,17 & $-0,19$ & $-0,15$ & $-6,38$ & $-4,97$ \\
\hline SNPAM & 101,2 & 156,11 & 0,26 & 0,17 & 17,62 & 13,29 \\
\hline SODA & 1002,4 & 84,82 & 0,16 & 0,13 & 5378,39 & 4862,27 \\
\hline SODSN & 25,1 & 105,53 & 0,29 & 0,28 & 13,05 & 13,37 \\
\hline SOKM & 1161,7 & 33,16 & $-0,07$ & $-0,05$ & $-23,17$ & $-23,09$ \\
\hline SONME & 66,9 & 167,11 & 1,44 & 0,10 & 18,16 & 2,23 \\
\hline SRVGY & 127,7 & 168,20 & 0,09 & 0,05 & 14,38 & 8,45 \\
\hline SUMAS & 11,7 & 104,79 & 0,27 & 0,25 & 6,16 & 6,35 \\
\hline TACTR & 6,1 & 67,51 & $-0,16$ & $-0,18$ & $-7,19$ & $-7,22$ \\
\hline TATGD & 193,4 & 185,28 & 0,06 & 0,06 & 17,39 & 19,27 \\
\hline TAVHL & 902,4 & 86,39 & 0,14 & 0,09 & 2222,92 & 1680,57 \\
\hline TBORG & 707,6 & 83,82 & 0,18 & 0,17 & 9624,26 & 9631,18 \\
\hline TCELL & 5157,2 & 86,78 & 0,08 & 0,07 & 9364,74 & 8680,02 \\
\hline TDGYO & 21,5 & 97,23 & 0,09 & 0,11 & 15,63 & 15,57 \\
\hline TEKTU & 78,2 & 10,91 & $-0,02$ & $-0,04$ & $-10,45$ & $-17,06$ \\
\hline TGSAS & 16,6 & 61,21 & 0,00 & 0,00 & 8,55 & 8,57 \\
\hline THYAO & 2378,5 & 83,03 & 0,08 & 0,03 & 3119945,99 & 1472180,90 \\
\hline TIRE & 189,4 & 21,63 & $-0,02$ & $-0,02$ & $-69,80$ & $-62,34$ \\
\hline TKFEN & 921,8 & 85,10 & 0,12 & 0,11 & 3876,51 & 3639,23 \\
\hline TKNSA & 106,6 & 82,49 & $-0,13$ & $-0,10$ & $-4267,33$ & $-4263,28$ \\
\hline TKURU & 2,3 & 79,64 & $-0,05$ & $-0,17$ & $-11,47$ & $-37,37$ \\
\hline TLMAN & 41,3 & 189,67 & 0,40 & 0,38 & 4,72 & 4,75 \\
\hline TMPOL & 15,3 & 109,46 & 0,08 & 0,15 & 5,91 & 11,04 \\
\hline TMSN & 114,8 & 48,30 & 0,34 & $-0,03$ & $-19,88$ & 2,06 \\
\hline
\end{tabular}

Copyright (C) 2021 by IJSSER

ISSN: 2149-5939 


\begin{tabular}{|c|c|c|c|c|c|c|}
\hline TOASO & 1850,3 & 85,21 & 0,12 & 0,12 & 7430,48 & 7326,56 \\
\hline TRCAS & 132,2 & 12,70 & $-0,03$ & $-0,02$ & $-27,43$ & $-19,51$ \\
\hline TRGYO & 528,9 & 83,58 & 0,07 & 0,06 & 3635,32 & 3635,32 \\
\hline TRKCM & 821,3 & 83,45 & 0,10 & 0,05 & 6354,55 & 3721,52 \\
\hline TSGYO & 134,2 & 177,81 & 0,10 & 0,09 & 15,32 & 15,35 \\
\hline TSK,TSKB & 493,6 & 80,95 & 0,02 & 0,02 & 4185,76 & 3098,37 \\
\hline TSPOR & 214,9 & 9,48 & $-0,07$ & $-0,06$ & $-35,22$ & $-34,89$ \\
\hline TTKOM & 4028,4 & 84,64 & 0,05 & 0,06 & 9958,93 & 12049,25 \\
\hline TTRAK & 697,1 & 233,27 & 0,04 & 0,04 & 37,07 & 36,98 \\
\hline TUCLK & 23,3 & 78,14 & 0,02 & 0,01 & 86,30 & 27,09 \\
\hline TUKAS & 344,8 & 289,23 & 0,15 & 0,13 & 19,14 & 19,39 \\
\hline TUPRAS & 3117,6 & 81,29 & 0,15 & 0,00 & 316910,46 & 2655,82 \\
\hline TURGG & 57,2 & 81,28 & 0,01 & 0,01 & 146,50 & 146,81 \\
\hline UFUK & 36,2 & 66,30 & 0,29 & $-0,02$ & $-25,27$ & 2,50 \\
\hline ULAS & 7,6 & 77,00 & $-0,03$ & $-0,03$ & $-42,73$ & $-41,27$ \\
\hline ULKER & 1262,6 & 84,15 & 0,11 & 0,08 & 7429,09 & 5988,42 \\
\hline ULUSE & 451,1 & 262,35 & 0,20 & 0,17 & 29,67 & 30,12 \\
\hline ULUUN & 111,9 & 97,33 & 0,03 & 0,02 & 24,66 & 22,34 \\
\hline UMPAS & 4,6 & 10,94 & $-0,21$ & $-0,28$ & $-0,44$ & $-0,49$ \\
\hline USAK & 58,2 & 71,40 & 0,01 & 0,01 & 32,50 & 39,65 \\
\hline UTPYA & 23,5 & 49,51 & 0,00 & $-0,01$ & $-27,36$ & $-93,79$ \\
\hline UZERB & 5,8 & 80,32 & $-0,09$ & $-0,08$ & $-50,17$ & $-48,54$ \\
\hline VAKBN & 2771,1 & 89,33 & 0,38 & 0,24 & 5883,77 & 3757,26 \\
\hline VAKFN & 70,4 & 82,76 & 0,01 & 0,01 & 13264,93 & 11546,39 \\
\hline VAKKO & 117,7 & 223,59 & 0,08 & 0,07 & 8,35 & 8,78 \\
\hline VANGD & 6,2 & 56,80 & $-0,24$ & $-0,27$ & $-3,94$ & $-3,93$ \\
\hline VERTU & 41,1 & 144,84 & 0,18 & 0,17 & 7,84 & 7,84 \\
\hline VERUS & 280,5 & 234,37 & 0,18 & 0,15 & 22,06 & 20,73 \\
\hline VESBE & 742,4 & 83,85 & 0,12 & 0,11 & 7775,92 & 7867,18 \\
\hline VESTL & 799,6 & 81,06 & 0,03 & 0,00 & 135217,28 & 9729,26 \\
\hline VKFYO & 24,8 & 83,67 & 0,06 & 0,06 & 125,83 & 124,99 \\
\hline VKGYO & 191 & 218,83 & 0,06 & 0,05 & 11,82 & 11,81 \\
\hline VKING & 28,1 & 9,33 & $-0,08$ & $-0,12$ & $-4,99$ & $-7,33$ \\
\hline YAPRK & 10,7 & 83,34 & 0,04 & 0,04 & 35,12 & 35,12 \\
\hline YATAS & 178,7 & 238,39 & 0,10 & 0,09 & 12,33 & 12,59 \\
\hline YAYLA & 21,7 & 87,43 & 0,17 & 0,02 & 71,61 & 6,53 \\
\hline YBTAS & 101,3 & 57,38 & $-0,04$ & $-0,05$ & $-77,58$ & $-77,05$ \\
\hline YESIL & 15,6 & 186,01 & 0,17 & 0,15 & 1,78 & 1,78 \\
\hline YGGYO & 280,6 & 752,51 & 0,16 & 0,15 & 4,87 & 4,85 \\
\hline YGYO & 32,2 & 15,77 & $-0,05$ & $-0,05$ & $-1,62$ & $-1,62$ \\
\hline YKBNK & 2818,2 & 71,04 & 0,01 & 0,01 & 4657,62 & 7686,27 \\
\hline YKGYO & 21,4 & 86,48 & 0,04 & 0,04 & 36,28 & 37,09 \\
\hline YKSLN & 71,9 & 116,01 & 0,11 & 0,09 & 23,74 & 23,80 \\
\hline YONGA & 60,9 & 82,17 & 0,01 & 0,02 & 215,77 & 674,19 \\
\hline YUNSA & 67,6 & 79,60 & 0,01 & 0,02 & 79,88 & 116,63 \\
\hline YYAPI & 33,2 & 9,36 & $-0,07$ & $-0,07$ & $-4,91$ & $-4,91$ \\
\hline ZOREN & 740,1 & 80,21 & $-0,04$ & $-0,01$ & $-27883,91$ & $-6035,81$ \\
\hline
\end{tabular}

The ratio of period net profit to total assets used in profitability analysis shows how profitable companies are according to their total assets. In the graphs comparing Turkish companies and Forbes 2000 companies, changes in the period coefficients have been observed and these differences are shown in Figure 4. Although there are companies that use their investments efficiently from companies in Borsa Istanbul, their inefficiency rates appear to be significantly higher than Forbes companies. In line with the data obtained, the reason for the lower investment returns of Turkish companies seems to be the inability to generate profit. When this situation in Turkish companies is revised, companies can be in a position to earn more with less investment. The column chart version of the asset profitability differences of Borsa İstanbul companies and Forbes 2000 companies is given in figure 5. 


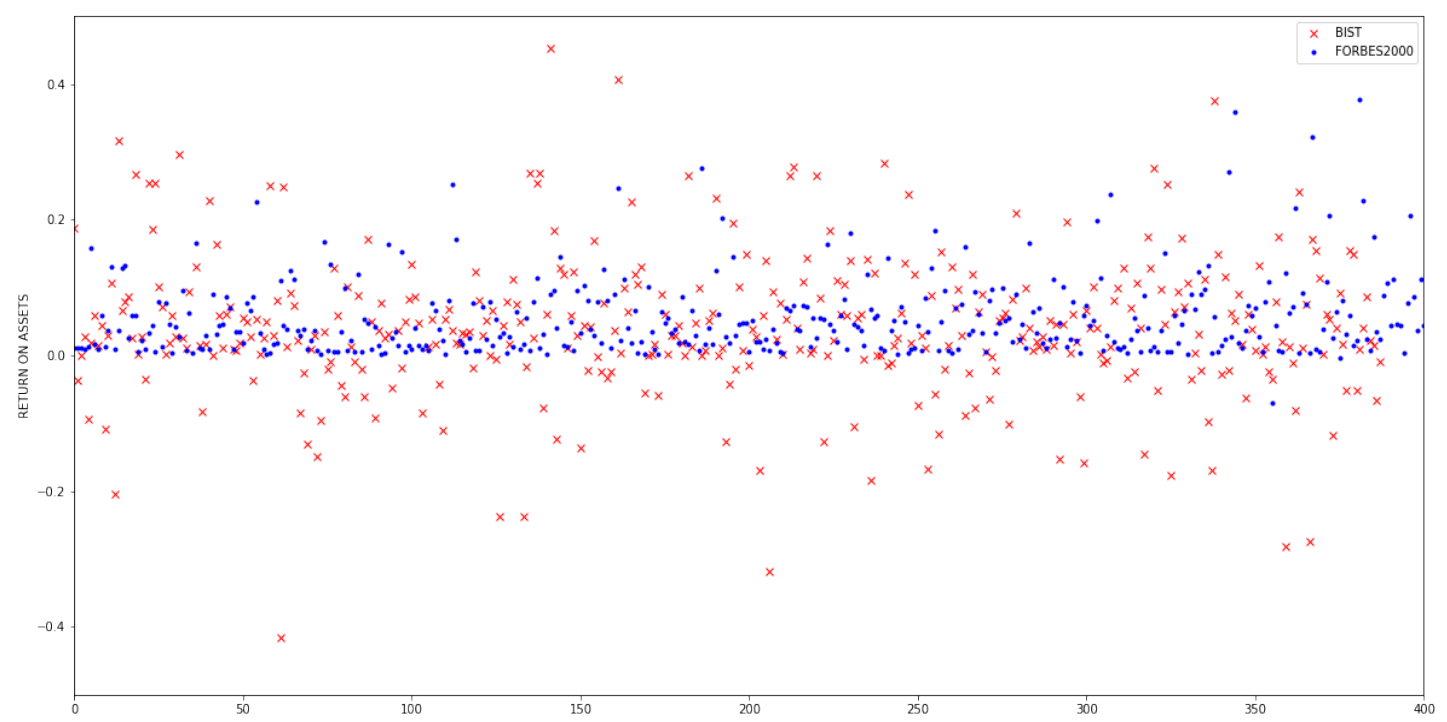

Figure 4. Asset profitability distribution graph of Borsa İstanbul and Forbes 2000 Firms

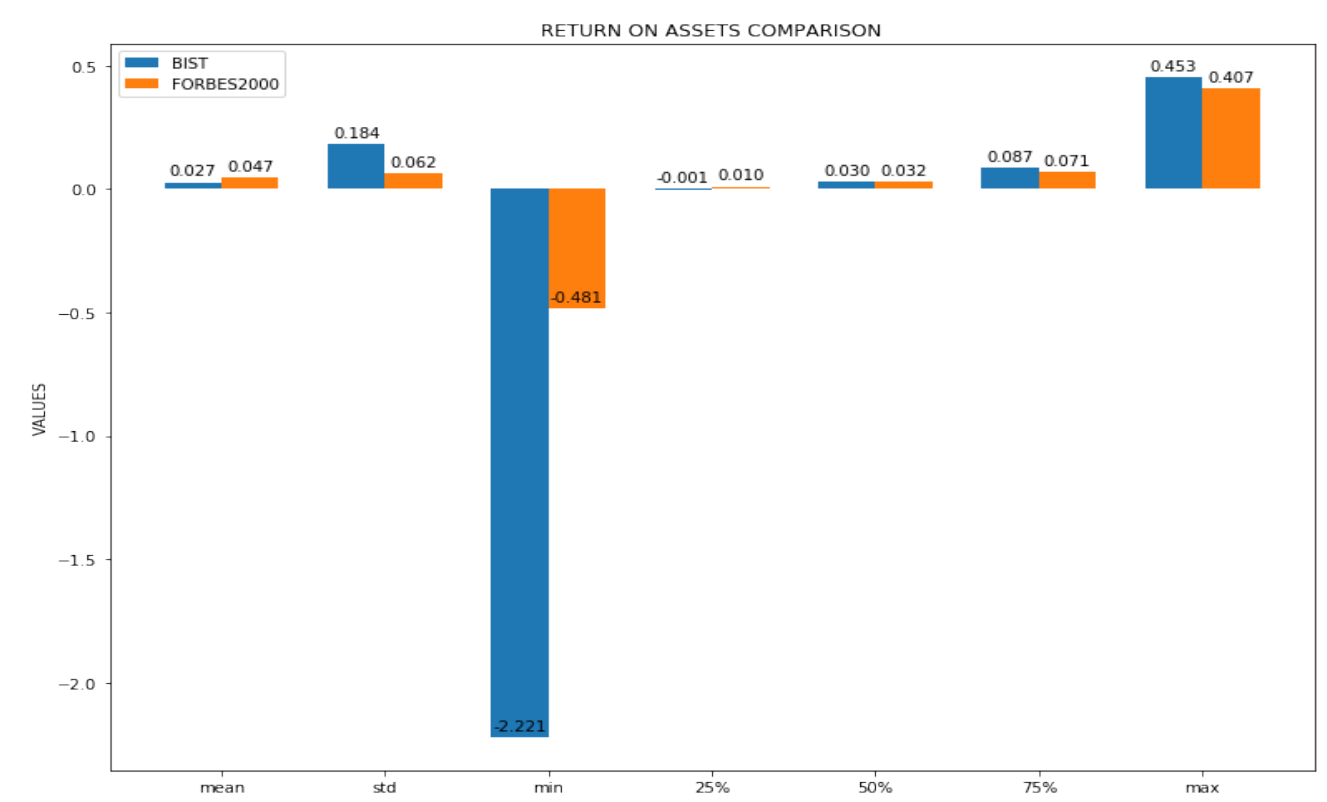

Figure 5. Asset profitability column chart of Borsa İstanbul and Forbes 2000 Firms

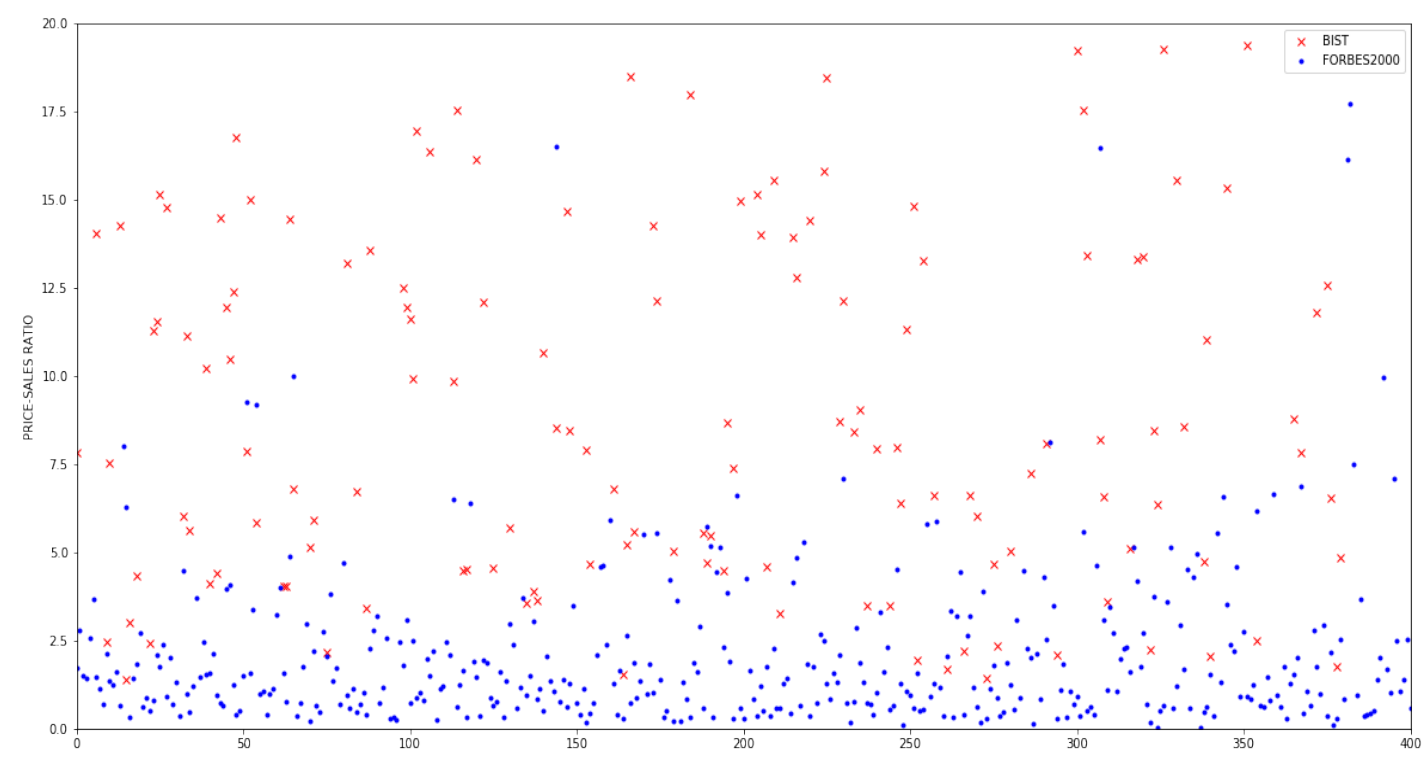

Figure 6. Price sales ratio distribution chart of Borsa İstanbul and Forbes 2000 Firms 
The price / sales ratio indicating how many times the company value is the business volume and how much money companies will pay for each unit of sales are found. When the graphs of Turkish companies and companies in Forbes 2000 are examined, it is seen that the ratio of Forbes companies is smaller. If this ratio is small, it indicates a positive situation but a high does not mean that the company is cheap. However, when Borsa İstanbul companies are analyzed by sector, it is concluded that some companies are smaller than their multiplier averages. These differences are shown in Figure 6. It has been concluded that companies with low price / sales ratio, which is very important for investors, make future pricing or company shares are quite expensive. With future pricing, which is a risky situation, companies promise that they will make higher profits in the future than their current profits. In this case, it is necessary to pay attention to whether there is manipulation or not and the relevant institutions should follow this situation. The column chart of the price-selling ratio differences between Borsa Istanbul companies and Forbes 2000 companies is given in Figure 7.

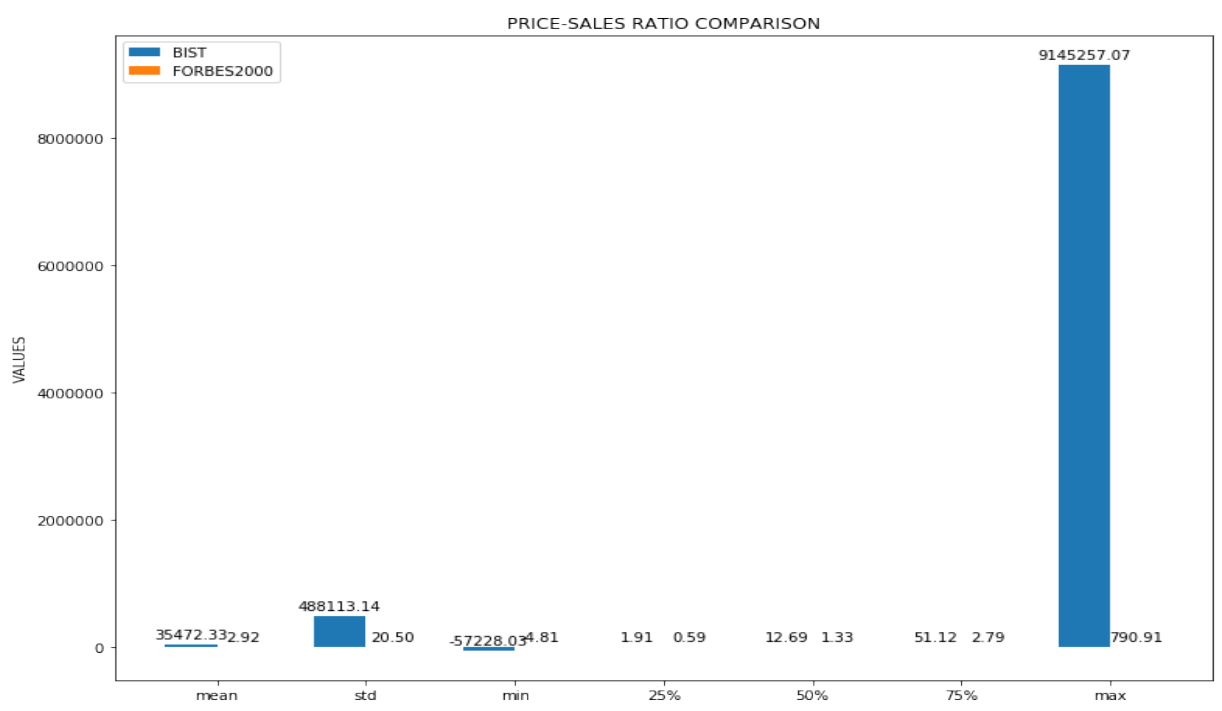

Figure 7. Price sales course column chart of Borsa İstanbul and Forbes 2000 Firms

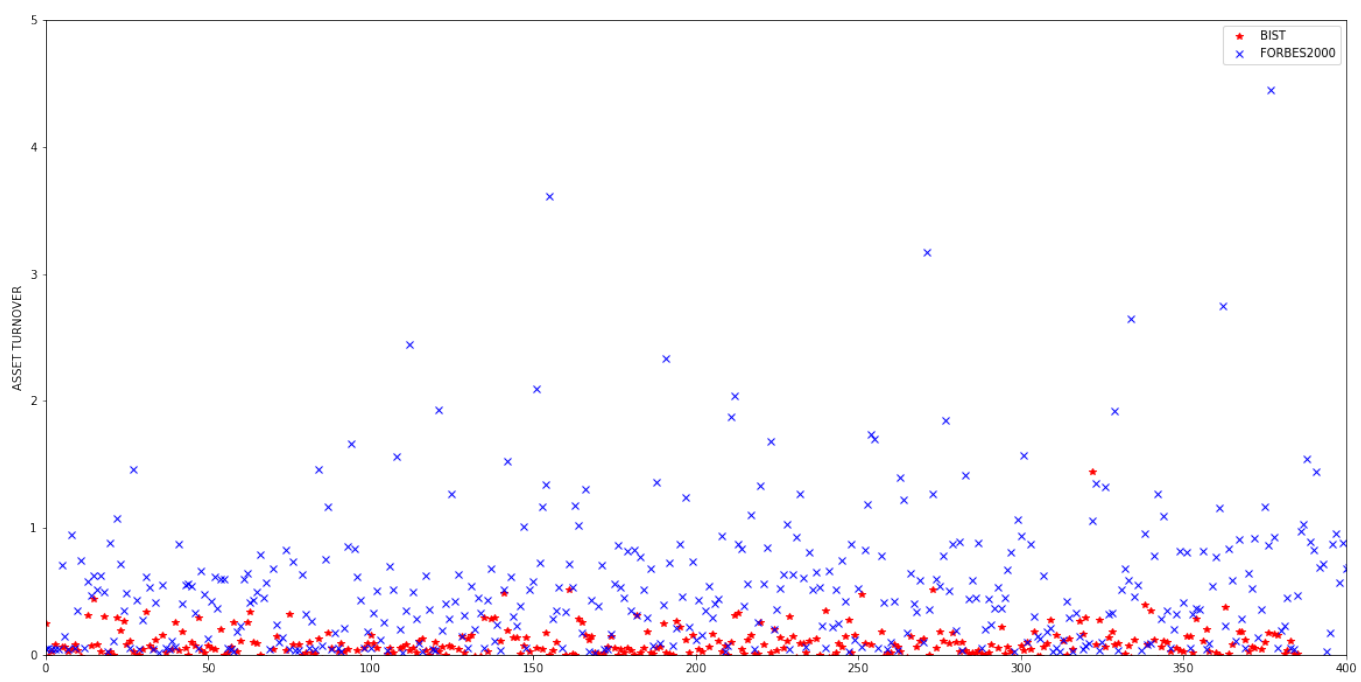

Figure 8. Asset Turnover Distribution Point Graph of Borsa İstanbul and Forbes 2000 Firms

Turnover ratio of assets (actives) is a kind of risk measure. The ratio is obtained by the formula of proportioning annual sales to total assets. As it can be understood from the formula, increasing the turnover of the company or decreasing the balance sheet will increase the asset turnover rate. It can be said that there are idle resources in companies that have the effect of reducing the turnover rate. The higher turnover of the companies listed in Forbes 2000 in terms of million $\$$ in the charts than the companies in Borsa Istanbul indicates that the companies have less risk factor. These differences are shown in Figure 8. Although the ratio varies from sector to sector, the fact that this ratio is 1.5 and above indicates that the company is doing enough. It can be said that the situation of companies with 2 and above is quite good. Based on the graphics, it can be said that companies in Borsa Istanbul have lower asset turnover rates than Forbes 2000 companies. The column chart of the asset turnover differences between Borsa İstanbul companies and Forbes 2000 companies is given in Figure 9. 


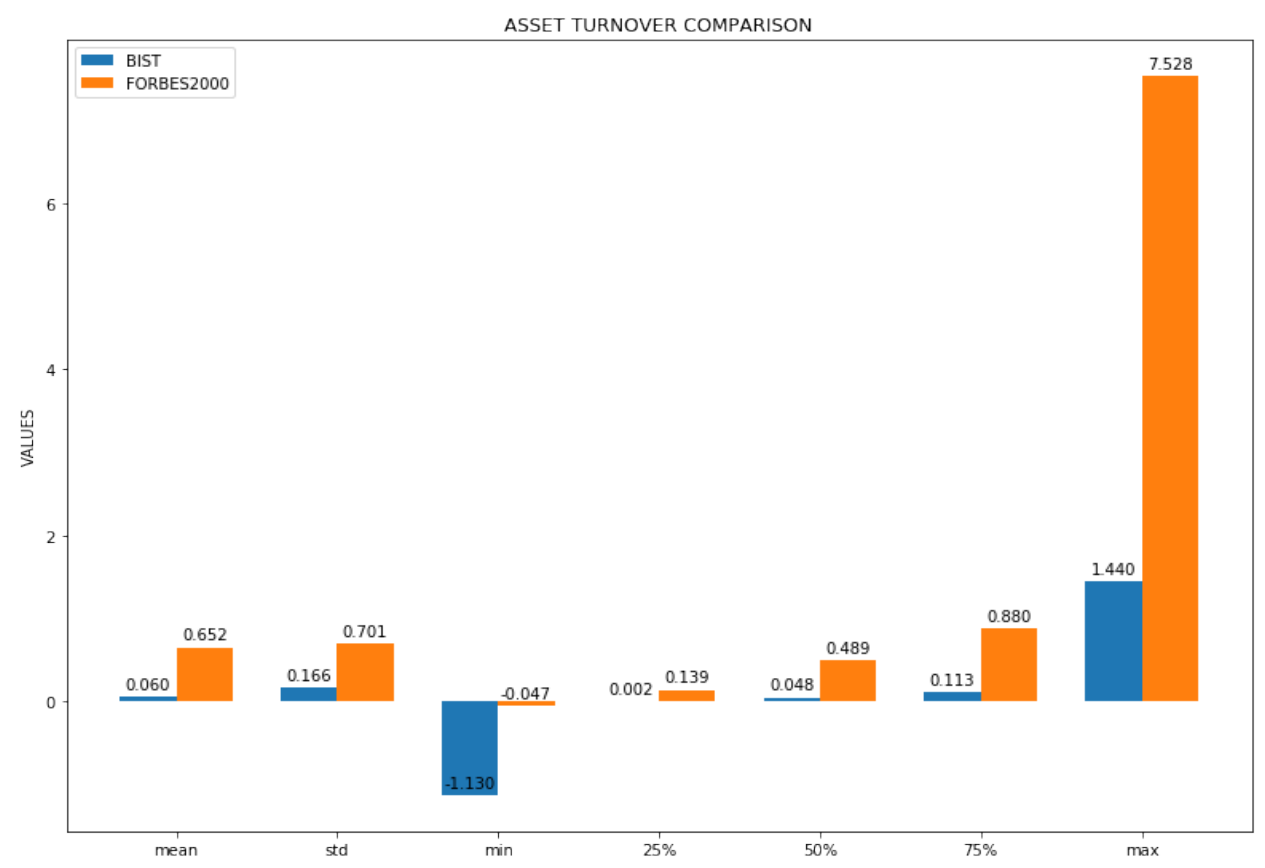

Figure 9. Asset turnover column chart of Borsa İstanbul and Forbes 2000 Firms

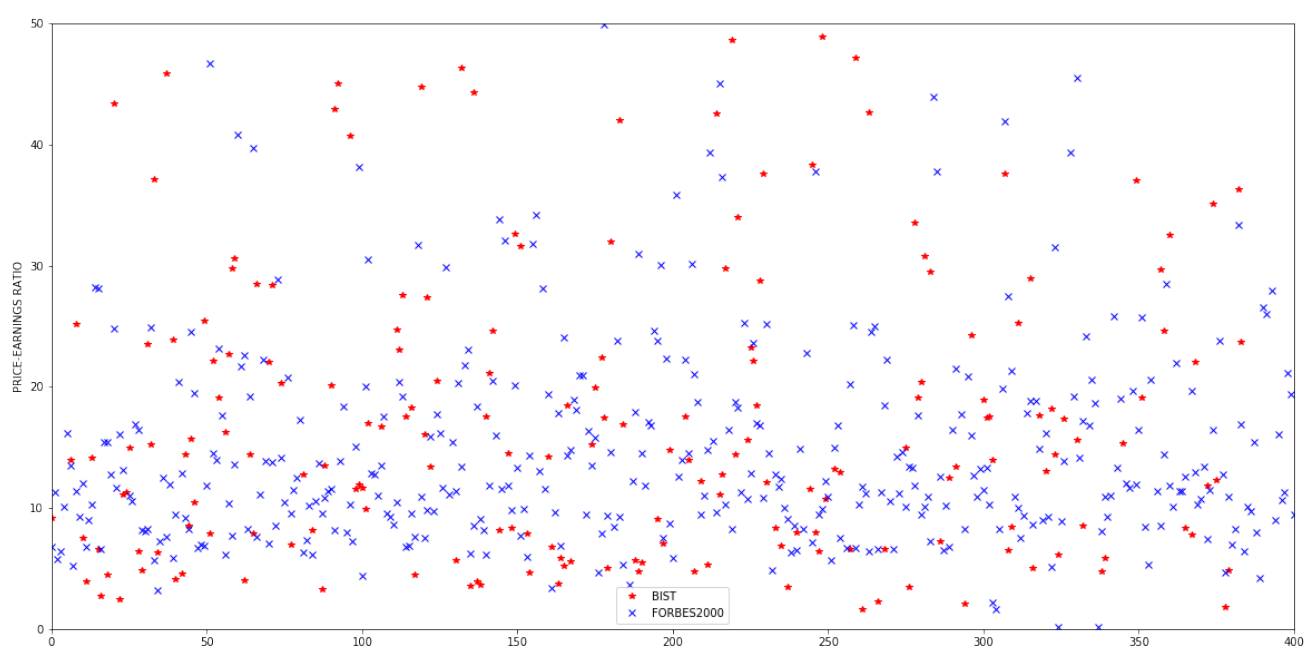

Figure 10. Distribution graph of stock price measurement of Borsa İstanbul and Forbes 2000 Firms

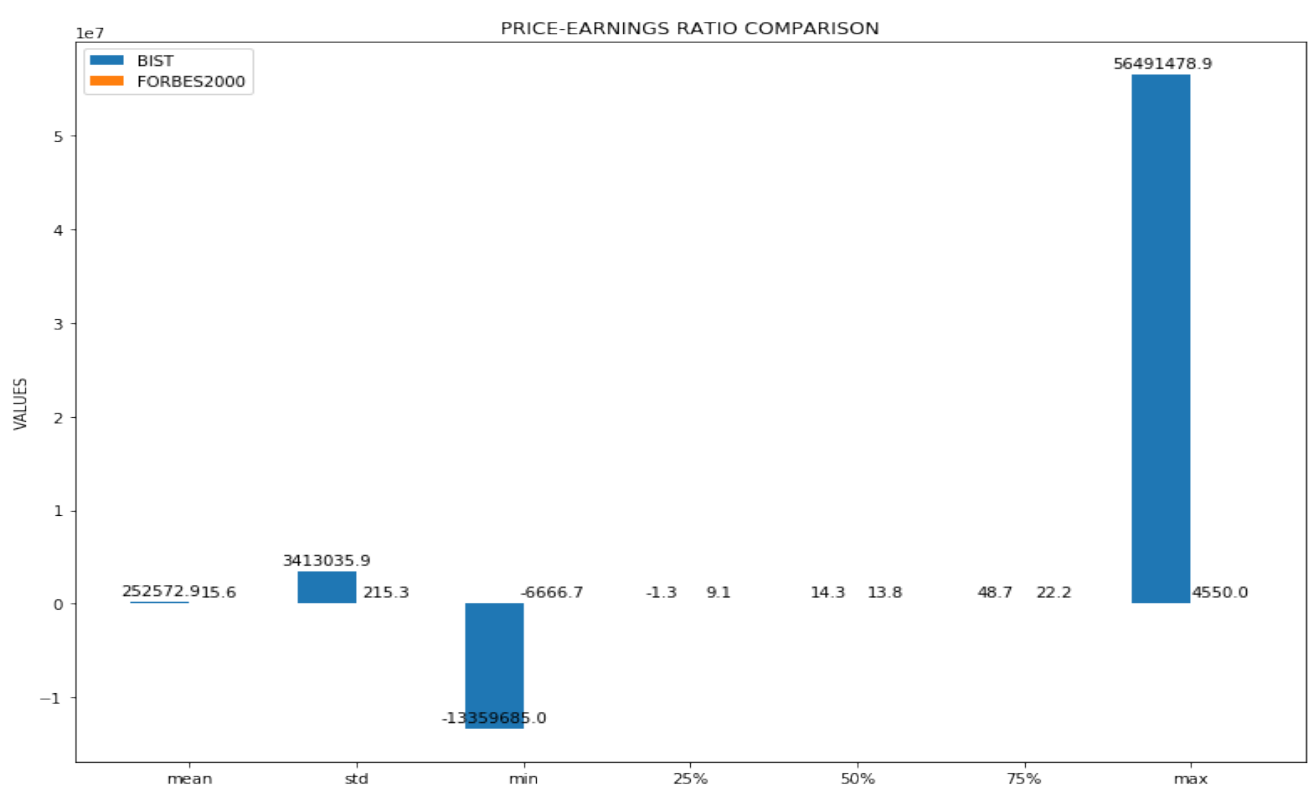

Figure 11. Column chart of stock price measurement distribution of Borsa İstanbul and Forbes 2000 Firms 
The analysis performed is within the scope of fundamental analysis and is based on estimating the value of a stock or the entire market. The point distribution graph of the share price measurement ratio differences of Borsa İstanbul companies and Forbes 2000 companies is given in Figure 10. Share price measurement is one of the financial ratios frequently used by both shareholders and managers. The fact that the rates of Turkish companies are higher than they should be is a proof that there are directions within the company and they can be easily affected by possible exchange rate fluctuations. This will create a particularly negative situation for small investors. Investors will continue to suffer as long as they are not examined by the necessary institutions and penal sanctions are imposed. The column chart of the share price measurement ratio differences of Borsa İstanbul firms and Forbes 2000 firms is given in Figure 11. A sectoral analysis reveals that the real market values of the Factoring and Banking sectors are quite high and have a rate-increasing effect. Among the sectors, the companies that use their assets in the best way have been mining / quarry and chemical product enterprises. While it is a good situation to have companies with high ratios, having low sectors can be a dangerous situation. It was observed that the Factoring and Construction companies made a more profitable investment on a sectoral basis, from the rate at which the companies were calculated how much money would be paid for each unit of sales. Figure 12 shows real market value, active profitability in Figure 13, and sectoral differences of price sales ratios in Figure 14.

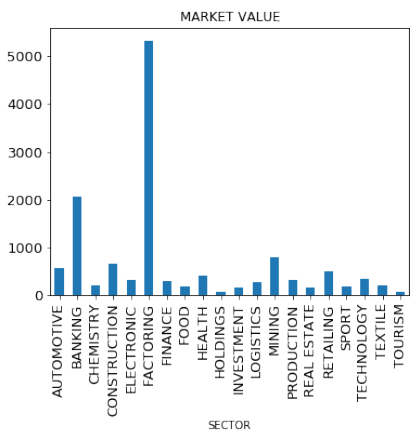

Figure 12. Market value chart

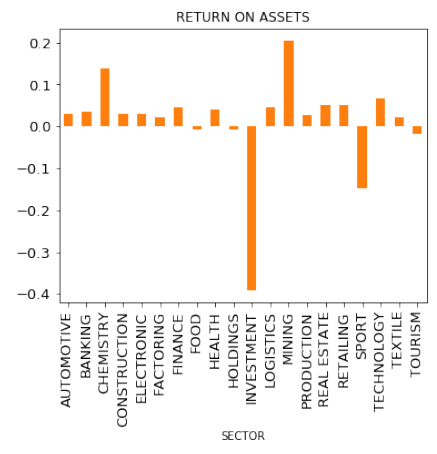

Figure 13. Active profitability chart

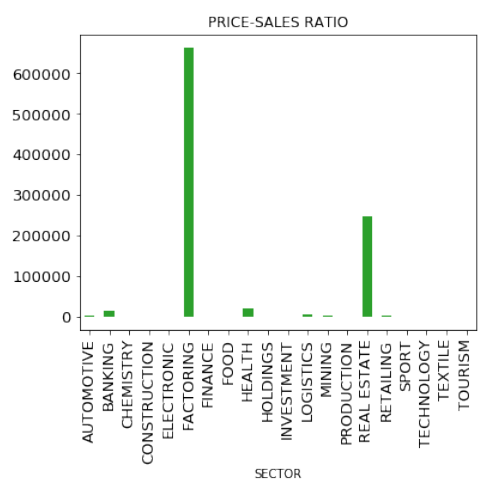

Figure 14. Sectoral comparison charts for price sales ratios

It is seen that the difference between the actual value created by the Turkish companies in Borsa Istanbul and the estimated value emerging in the software is quite high. As a result of the analysis, this situation is acceptable and these ratios that give clues about the real and estimated market values of the companies are given in Figure 17.

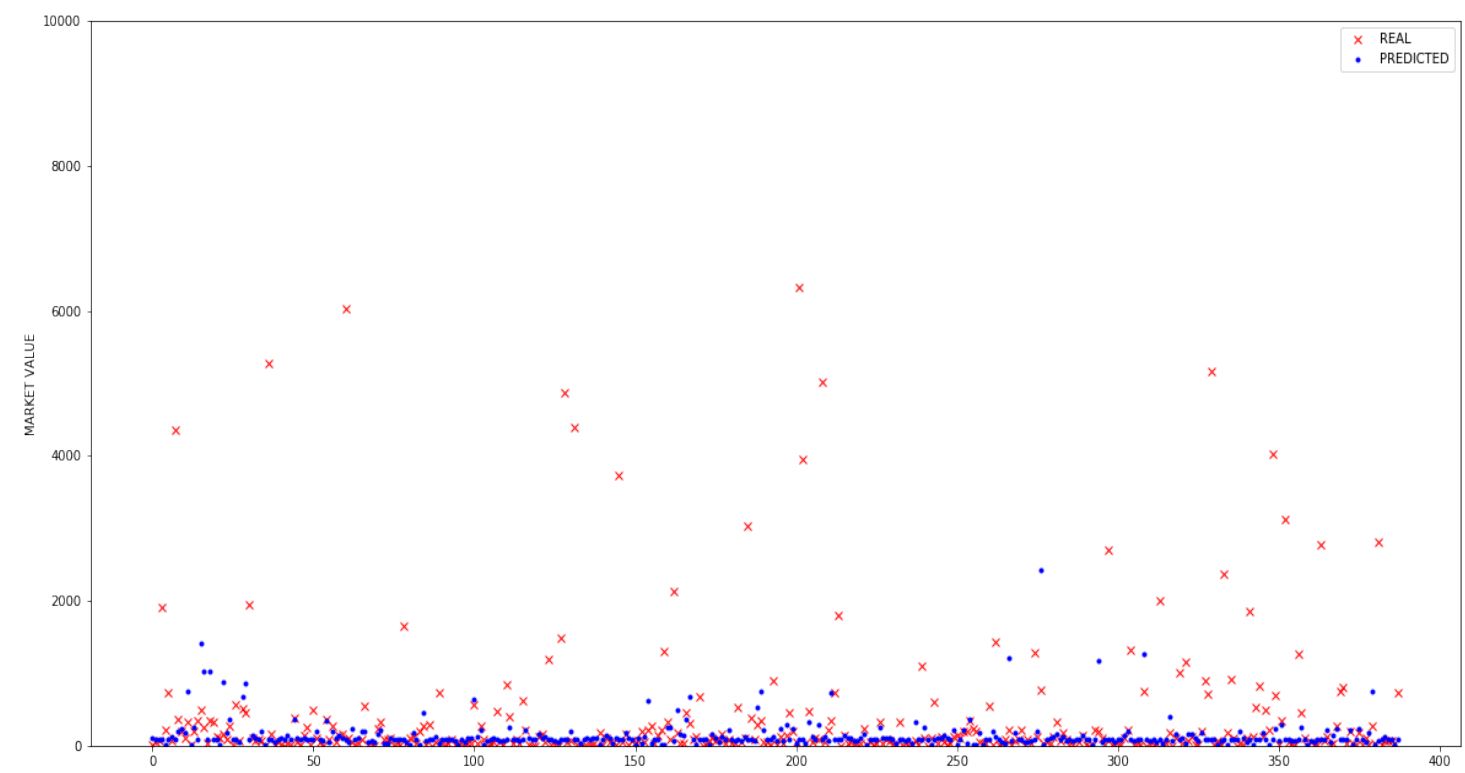

Figure 17. Comparison chart for real and estimated market values of Borsa İstanbul Firms

When the possible reasons for this difference seen in the chart are investigated, it can be concluded that the company shares are at a very high level of what they should be, the asset management is not carried out well and the shares of the company that are traded in the stock exchange are exposed to manipulative effect. In addition, it is an inevitable end that the company shares in this situation will fall sharply in possible crises. In order to prevent 
this situation, the control over the stocks should increase and the win-lose situation should be replaced by a winwin policy. Column chart showing the differences between actual and estimated values is given in Figure 18.

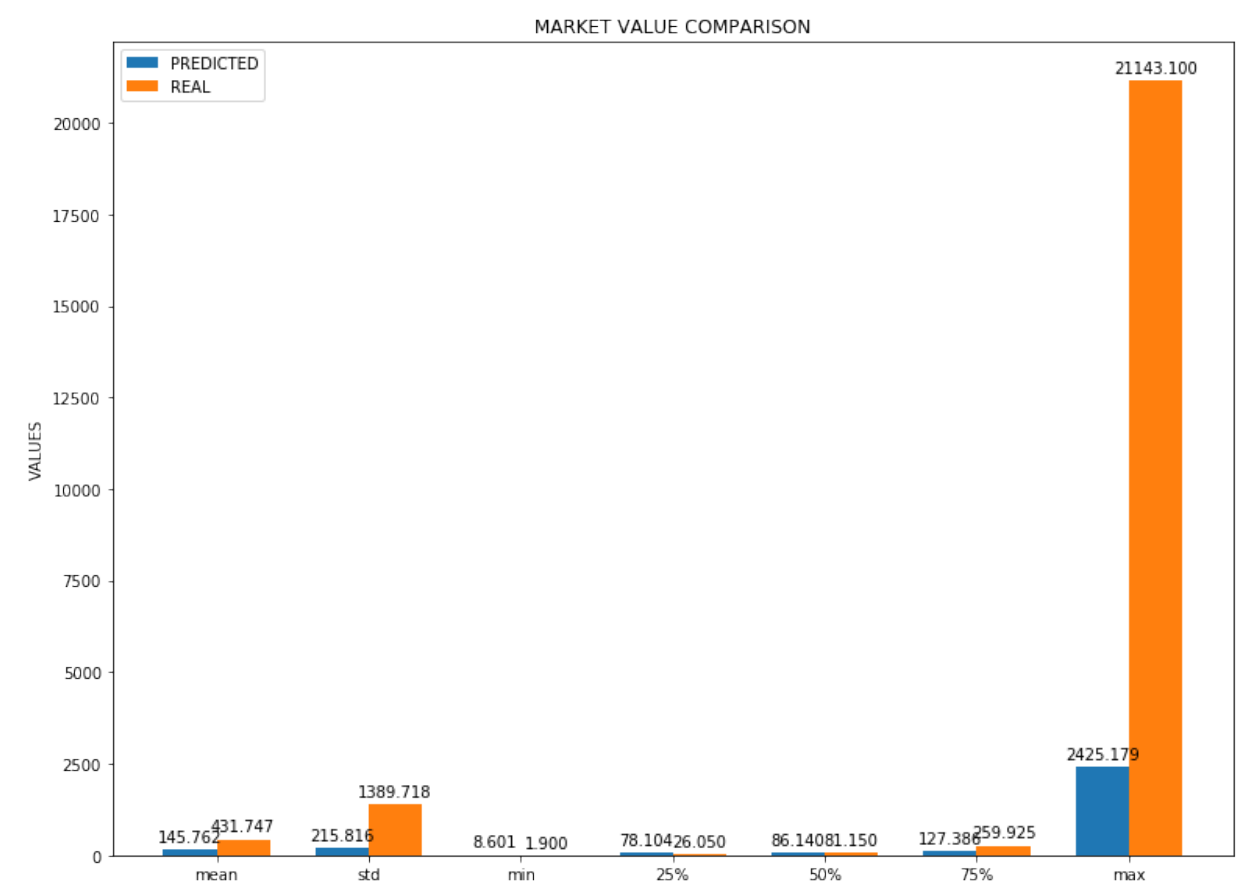

Figure 18. Comparison of real and estimated market values of Borsa İstanbul firm's column chart

When the correlation table of the ratio analysis is examined, the relationship between the real market value and the stock price measurement is at a moderate level, while the price selling ratio is at a high level close to the level of perfection, the relationship between the other variables and the real market value is weak or 0 . On the other hand, there is an excellent relationship between the price selling ratio and the share price measurement plot and they are highly affected by each other. If the asset is profitability, there are 3 variables and it is weakly related to variables such as profit / loss, estimated market value and asset turnover. The ratio in 2019 sales is moderate with the estimated market value, while there is a zero or no relationship with 2018 assets and 2019 profit / loss with other weak variables. In the case of profit and loss, there is a high relationship with the estimated market value, a moderate relationship with 2019 and 2018 assets, while there is a weak relationship between return on assets and 2019 sales. The strongest relationship in predicted market value is affecting the profit / loss situation of 2019, 2019 sales, 2019 assets and 2018 assets moderately affecting this value. The assets that affect 2019 the most are 2018 assets at the level of excellence with 0.99 . The only variable in asset profitability can be mentioned, and this is the weak relationship between 2019 sales. The correlation graph is shown in Figure 19.

\begin{tabular}{|c|c|c|c|c|c|c|c|c|c|c|}
\hline MARKET VALUE & 1.00 & 0.25 & 0.05 & 0.04 & 0.04 & -0.01 & -0.01 & -0.02 & -0.02 & -0.03 \\
\hline PRICE-SALES RATIO & 0.25 & 1.00 & -0.01 & -0.02 & 0.15 & -0.01 & -0.02 & -0.02 & -0.03 & -0.03 \\
\hline RETURN ON ASSETS & 0.05 & -0.01 & 1.00 & 0.79 & -0.01 & 0.22 & 0.20 & 0.15 & 0.00 & -0.00 \\
\hline ASSET TURNOVER & 0.04 & -0.02 & 0.79 & 1.00 & -0.02 & 0.21 & 0.22 & 0.28 & -0.03 & -0.04 \\
\hline PRICE-EARNINGS RATIO & 0.04 & 0.15 & -0.01 & -0.02 & 1.00 & -0.02 & -0.02 & -0.02 & -0.03 & -0.03 \\
\hline PROFIT(2019) & -0.01 & -0.01 & 0.22 & 0.21 & -0.02 & 1.00 & 0.87 & 0.69 & 0.49 & 0.46 \\
\hline REDICTED MARKET VALUE & -0.01 & -0.02 & 0.20 & 0.22 & -0.02 & 0.87 & 1.00 & 0.82 & 0.57 & 0.54 \\
\hline REVENUE(2019) & -0.02 & -0.02 & 0.15 & 0.28 & -0.02 & 0.69 & 0.82 & 1.00 & 0.41 & 0.38 \\
\hline ASSET(2019) & -0.02 & -0.03 & 0.00 & -0.03 & -0.03 & 0.49 & 0.57 & 0.41 & 1.00 & 0.99 \\
\hline ASSET(2018) & -0.03 & -0.03 & -0.00 & -0.04 & -0.03 & 0.46 & 0.54 & 0.38 & 0.99 & 1.00 \\
\hline & 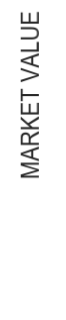 & 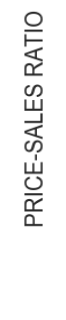 & 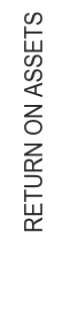 & 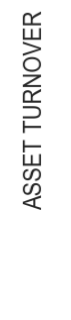 & 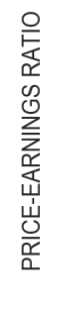 & 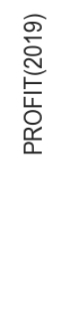 & 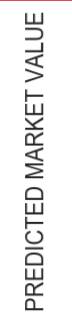 & 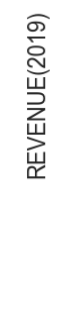 & 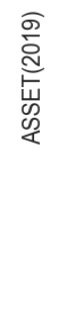 & 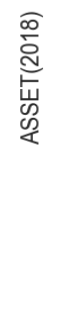 \\
\hline
\end{tabular}

Figure 19. Correlation matrix 


\section{Conclusion}

In this research, the data of the best 2000 companies in the world according to Forbes were taken as optimum and the estimated market values of Turkish companies in Borsa Istanbul were obtained. According to the research, the market value of 235 of 388 Turkish firms was higher than the estimated market value, while the market value of 153 firms was below the estimated values. To support these results and to understand where the problem lies, ratio analyzes were made and compared with Forbes 2000 companies and Borsa İstanbul companies.

The first thing that stands out in ratio analysis is the asset transfer results. According to Forbes 2000 data, none of the Borsa Istanbul companies is above 1.5. In this case, it can be said that Borsa İstanbul companies do not use their assets at $100 \%$ capacity or that the idle capacity is high. It is seen that the value of textile and mining enterprises among these companies is high. Likewise, the company with the closest value to 1.5 is SÖNMEZ textile company. In order to understand whether the investments made by the companies in general are correct or not, the return on assets ratios, which show how efficiently the companies are invested according to their total assets, have been determined.

It was determined that 209 of the companies were above the general average of 0.03 according to their return on assets. However, these data should be based on the industry average. Only 209 of Borsa Istanbul companies are above the general average, and 179 are below the general average. Sectorally, mining is the most productive investment sector. In the analysis of both the asset turnover rate and the return on assets ratio, it is seen that some Borsa Istanbul companies keep this amount as investment amount instead of distributing it as dividend, although they make a profit. However, it can be said that this investment, which is spent instead of dividends, is not efficient enough due to idle capacities.

There are 2 other ratio analyzes to get information about market values, namely price to sale ratio and share price measurement. According to share price measurements, 380 companies are below the general average. According to the price-sales ratio, 381 companies are above the general average. These ratios should be lower than the industry average, not the general. The clearest result that can be said as a result of share price measurement and price sales ratio analysis is that banks and factoring companies make excessive profits according to their firm values. Because there is little need for fixed assets in both sectors, it can keep assets and firm value lower than profits.

Borsa Istanbul is the only market in which Turkey has shares. The Turkish economy, which is important for foreign investors in terms of markets, can be affected by small fluctuations in exchange rates and Borsa Istanbul indices can be affected either extremely negatively or extremely positively. According to the analysis, although these firms have high potential, they do not seem profitable enough. For this reason, Borsa İstanbul companies should be well supervised by state authorities and investors should be able to trade on more transparent data.

In the study, the ratio can be calculated on the basis of company data and in general, but researchers who want to work on this subject can reach comparative information about companies and markets by reaching ratio averages on a sectoral basis or they can reach stronger results, for example, they can detail the research by accessing equity information. Valuable investors who will invest in Borsa Istanbul are recommended to invest using the methods shown in the research, to examine the balance sheets, cash flow statements and income statements of companies, and to invest by taking into account the country's economy. In this way, both the information about how to invest in a company and the extent to which the company will be affected by possible manipulative movements will be known in advance.

\section{Author contribution statements}

Authors contributed equally to the design and implementation of the research, to the analysis of the results and to the writing of the manuscript.

\section{Disclosure statement}

No potential conflict of interest was reported by the authors.

\section{Ethics committee approval}

Ethics Committee Approval is not required for this study. All responsibility belongs to the researchers. 


\section{References}

Aggarwal, R. K., \& Wu, G. (2003, March). Stock market manipulation-theory and evidence. In AFA 2004 San Diego Meetings. Angenieux, G. (1964). Les Ratios et L’Expansiyon de L’Entreprise. Paris: Dunod.

Atalay, M., \& Çelik, E. (2017). Büyük Veri Analizinde Yapay Zekâ Ve Makine Öğrenmesi Uygulamalari-Artificial Intelligence and Machine Learning Applications in Big Data Analysis. Mehmet Akif Ersoy Üniversitesi Sosyal Bilimler Enstitüsü Dergisi, 9(22), 155-172.

Barnes, P. (2016). Stock market efficiency, insider dealing and market abuse. CRC Press.

Berman, K., Knight, J., \& Case, J. (2008). Financial intelligence for entrepreneurs: What you really need to know about the numbers. Harvard Business Press.

Bhagat, S. and B. Black (2001): Board independence and long-term firm performance, Journal of Corporation Law 27, 231273.

Boudriga, \& Ghachem, (2016). Does US stock market react differently to rating announcements during crisis period? The case of the 2008 worldwide financial crisis. American Journal of Finance and Accounting, 4(3-4), 193-214.

Brigham, E. F., \& Houston, J. F. (2012). Fundamentals of financial management. Cengage Learning.

Brownlee, J. (2019, September 13). Introduction to Python deep learning with Keras. Retrieved from https://machinelearningmastery.com/introduction-python-deep-learning-library-keras/, Etişim Tarihi: 25.06.2020.

Cao, Q., Leggio, K. B., \& Schniederjans, M. J. (2005). A comparison between Fama and French's model and artificial neural networks in predicting the Chinese stock market. Computers \& Operations Research, 32(10), 2499-2512.

Chen, L., Qiao, Z., Wang, M., Wang, C., Du, R., \& Stanley, H. E. (2018). Which artificial intelligence algorithm better predicts the Chinese stock market. IEEE Access, 6, 48625-48633.

Çelik, S. (2018). Büyük Veri. Gece Kitaplığı. Ankara. ISBN: 978-605-288-811-7.

Dase, R. K., \& Pawar, D. D. (2010). Application of Artificial Neural Network for stock market predictions: A review of literature. International Journal of Machine Intelligence, 2(2), 14-17.

Drake, P. P., \& Fabozzi, F. J. (2012). Financial ratio analysis. Encyclopedia of Financial Models.

Eiteman, A., \& Stonehill, D. (1986). Multinational Business Finance. California: Addison Wesley Publishing.

Giacomino, D. E., \& Mielke, D. E. (1993). Cash Flows: Another Approach to Ratio Analysis. Journal of Accountancy, 55-57.

Gibbons, J. D. (1976). Nonparametric Methods for Quantitative Analysis. New York: Holt, Rinehart and Winston.

Gordan, R. (2000). Macroeconomics. Addison-Wesley.

Hiransha, M., Gopalakrishnan, E. A., Menon, V. K., \& Soman, K. P. (2018). NSE stock market prediction using deep-learning models. Procedia computer science, 132, 1351-1362.

Howell, C. D. (1992). Statistical Methods For Psychology. California: Duxbury Press.

Kara, Y., Boyacioglu, M. A., \& Baykan, Ö. K. (2011). Predicting direction of stock price index movement using artificial neural networks and support vector machines: The sample of the Istanbul Stock Exchange. Expert systems with Applications, 38(5), 5311-5319.

Khan, Z. H., Alin, T. S., \& Hussain, M. A. (2011). Price prediction of share market using artificial neural network (ANN). International Journal of Computer Applications, 22(2), 42-47.

Kızıl, A., Fidan, M., Kızıl, C., \& Keskin, İ. (2013). Türkiye Muhasebe ve Finansal Raporlama Standartları. İstanbul: Der Yayınları.

Krantz, M. (2016). Fundamental analysis for dummies. John Wiley \& Sons.

Machado, M. A. V., \& de Medeiros, O. R. (2013). Does the liquidity effect exist in the brazilian stock market?. Available at SSRN 2217941.

Mansfield, E. (1998). Essential Microeconomics. Norton \& Company.

Næs, T., Kvaal, K., Isaksson, T., \& Miller, C. (1993). Artificial neural networks in multivariate calibration. Journal of Near Infrared Spectroscopy, 1(1), 1-11.

Needles, B. E., Powers, M., \& Crosson, S. V. (2013). Financial and managerial accounting. Nelson Education.

Nikolai, L. A., Bazley, J. D., \& Jones, J. P. (2009). Intermediate Accounting (Book Only). Cengage Learning. 
Patel, M. B., \& Yalamalle, S. R. (2014). Stock price prediction using artificial neural network. International Journal of Innovative Research in Science, Engineering and Technology, 3(6), 13755-13762.

Pathmind (2020). A beginner's guide to neural networks and deep learning. Retrieved from https://wiki.pathmind.com/neuralnetwork.

Peterson \& Fabozzi, (1999). Analysis of financial statements (Vol. 54). John Wiley \& Sons.

Peterson, P. (2006). Financial Management and Analysis. New York: McGraw-Hill Inc.

Qiu, M., Song, Y., \& Akagi, F. (2016). Application of artificial neural network for the prediction of stock market returns: The case of the Japanese stock market. Chaos, Solitons \& Fractals, 85, 1-7.

Rouse, M. (2019, August 29). What is an artificial neural network (ANN)?. Retrieved from https://searchenterpriseai.techtarget.com/definition/neural-network.

Samuelson, P. A., \& William D, N. (1995). Economics. McGr Angenieux, G. (1964). Les Ratios et L'Expansiyon de L'Entreprise. Paris: Dunod.

Singh, R., \& Srivastava, S. (2017). Stock prediction using deep learning. Multimedia Tools and Applications, 76(18), 1856918584 .

Sexton, R. L. (2015). Exploring economics. Cengage Learning.

Shim, J. K., \& Siegel, J. G. (1988). Handbook of Financial Analysis,Forecasting \& Modeling. New Jersey: Prentice-Hall.

Spiegel, M. R. (1972). Theory and Problems of Statistics. New York: McGraw-Hill Book Company.

Taner B., Akaya G. C. (2004): Sermaye Piyasası, Faaliyet Alanı ve Menkul Kiymetler

Thomas, R., \& Gup, B. E. (2010). The valuation handbook: valuation techniques from today's top practitioners (Vol. 480). John Wiley \& Sons.

Tracy, A. (2012). Ratio analysis fundamentals: how 17 financial ratios can allow you to analyse any business on the planet. RatioAnalysis. net.

Vargas, M. R., De Lima, B. S., \& Evsukoff, A. G. (2017, June). Deep learning for stock market prediction from financial news articles. In 2017 IEEE International Conference on Computational Intelligence and Virtual Environments for Measurement Systems and Applications (CIVEMSA) (pp. 60-65). IEEE.

Willems, K. (2019). (Tutorial) KERAS tutorial: DEEP learning in PYTHON. Retrieved from https://www.datacamp.com/community/tutorials/deep-learning-python.

Yetis, Y., Kaplan, H., \& Jamshidi, M. (2014, August). Stock market prediction by using artificial neural network. In 2014 World Automation Congress (WAC) (pp. 718-722). IEEE.

Zhang, Y., \& Wu, L. (2009). Stock market prediction of S\&P 500 via combination of improved BCO approach and BP neural network. Expert systems with applications, 36(5), 8849-8854. 